\title{
Meta-Analysis
}

\section{e Nucleoplasty, a Minimally Invasive Procedure for Disc Decompression: A Systematic Review and Meta-analysis of Published Clinical Studies}

Philipp Maximilian Eichen, MD¹, Nils Achilles, MD, Volker König, PhD, Ralph Mösges, MD, $\mathrm{PhD}^{1}$, Martin Hellmich, PhD ${ }^{1}$, Bastian Himpe, $\mathrm{MD}^{2}$, and Rainer Kirchner, MD, $\mathrm{PhD}^{2}$

\begin{abstract}
From: ${ }^{1}$ Institute of Medical Statistics, Informatics and Epidemiology (IMSIE), University Hospital of Cologne, Kerpener Str. 62, 50937 Cologne, Germany; ${ }^{2}$ Section for Orthopedics, University Medical

Center Schleswig-Holstein, Ratzeburger Allee 160, 23538 Lübeck, Germany

Address Correspondence: Rainer Kirchner, MD, PhD. Ratzeburger Allee 160 23538 Lübeck - Germany E-mail:

rainer.kirchner@uk-sh.de

Disclaimer: Research was funded by an unrestricted scientific grant from ArthroCare (Deutschland) AG. Conflict of interest: Each author certifies that he or she, or a member of his or her immediate family, has no commercial association (i.e., consultancies, stock ownership, equity interest, patent/licensing arrangements, etc.) that might

pose a conflict of interest in connection with the submitted manuscript.

Manuscript received: 06-24-2013 Revised manuscript received: 11-07-2013

Accepted for publication: 12-18-2013

Free full manuscript: www.painphysicianjournal.com

Background: Nucleoplasty, based on Coblation $\AA$ technology, is a minimally invasive procedure used to decompress herniated discs. Reviews to date - exclusively systematic reviews - recommend nucleoplasty for treating chronic back pain, although with the restriction of limited to fair evidence. We therefore aimed to summarize and interpret our calculated results, where possible comprehensively and quantitatively, using statistical methods in the context of a meta-analysis supplementing a systematic review. In the process, the central question was to statistically determine whether, and to what extent, nucleoplasty can positively affect pain relief and functional mobility as well as lower the complication rate.

Objective: Newly published studies made it possible to conduct a meta-analysis of the visual analog scale (VAS), a measuring instrument used to determine pain intensity, and the Oswestry Disability Index (ODI), a scale that reflects the degree of impairment in percent. In addition to having clearly sound evidence for analyzing VAS/NPS data, the present, newly compiled meta-analysis was able to summarize VAS and ODI data quantitatively and to calculate a total complication rate for the first time. It was thereby possible to make a first comparison between nucleoplasty and conservative therapy (including epidural steroid injection).

Study Design: This meta-analysis examined all study data published in clinical trials involving the nucleoplasty procedure for plasma disc decompression.

Methods: A systematic search using the terms nucleoplasty and/or plasma disc decompression was conducted for literature listed in MEDLINE. Twenty-seven eligible studies (22 prospective trials and 5 retrospective trials) were included, and pooled analyses as well as various subgroup analyses (differentiation between cervical and lumbar disc herniations, comparisons with alternative treatments such as epidural steroid injection) were performed based on their data.

Results: Pain decreased from a baseline VAS value of 7.27 to 2.12 (postop/first day), 2.50 (one week), 2.70 (2 weeks), 3.23 (one month), 2.66 (6 weeks), 2.84 (3 months), 3.06 (6 months), 3.03 (12 months), 1.54 (18 months), and 3.69 (24 months) after nucleoplasty. The ODI value (baseline: 58.95) dropped to 28.60 (one week), 29.00 ( 2 weeks), 23.21 (one month), 30.00 (6 weeks), 18.30 (3 months), 22.54 (6 months), 24.43 (12 months), 12.82 (18 months), and 36.98 (24 months). Compared to baseline, significant pain reduction and improvement in functional mobility after nucleoplasty were observed at every time point. Nucleoplasty showed a total complication rate of $1.5 \%$, with the individual rates being $0.8 \%$ for cervical and $1.8 \%$ for lumbar nucleoplasty. Nucleoplasty was superior to conservative therapy at every time point and for all 3 included parameters, at some measurement time points even significantly.

Conclusions: Nucleoplasty reduces pain in the long term and improves patients' functional mobility. It is an effective, low-complication, minimally invasive procedure used to treat disc herniations.

Key words: Nucleoplasty, plasma disc decompression, (contained) herniated discs, visual analog scale (VAS), numeric pain scale (NPS), complication rate, Oswestry Disability Index (ODI), pain reduction

Pain Physician 2014; 17:E149-E173
\end{abstract}


ow back pain is defined as pain in the back beneath the costal arch and above the natal clefts, with or without radiation (1).

Low back pain is one of the most frequently occurring types of pain (1). According to one investigation conducted in the Federal Republic of Germany, the point prevalence for back pain was $37.1 \%$, one-year prevalence was $76.0 \%$, and life-time prevalence was $85.5 \%$. Prevalence declined as the level of education increased; some even regard education as the most important predictor for the occurrence of back pain (2). It appears certain that a person's social status (as measured by their education, occupation, and income) is related to the risk of back pain (3). People of low social status reported back pain in general and severe back pain in particular much more often compared to those having a high socioeconomic status (4).

Corresponding with these findings are workrelated circumstances linked to the risk for back pain, particularly biomechanical working conditions (e.g., carrying and lifting of heavy loads), vibrations, and unfavorable body postures while working (5). Contrary to popular belief, however, it appears that predominantly sedentary work poses no significant risk for low back pain (6).

Results similar to those observed in Germany can also be found in the USA: $26.4 \%$ of the American adult population reported having had back pain that lasted at least one day within the past 3 months. According to this study, too, the prevalence of back pain decreased as education levels and incomes increased (7).

Back pain occurs both in industrialized and developing countries. Overall, up to one-half of the working population experiences an episode of back pain at least once a year. Hence, back pain represents a leading cause of absence from work and results in considerable economic loss (8). Based upon this finding, it is said that a worldwide yearly equivalent of 800,000 DALYs (disability-adjusted life year = measure of overall disease burden, expressed as the number of years lost due to ill-health, disability or early death [9]) is lost. Up to $40 \%$ of cases of back pain are attributed to occupational stress; a preventive approach can therefore take place in this area (8).

It is estimated that in the USA alone at least 116 million adults suffer from chronic pain, incurring annual costs of 560 to 635 billion US dollars resulting from medical treatment and production losses (10).

Thus, in the US health care system the annual costs of pain were higher than expenses for heart disease, cancer, and diabetes (11). As for the European Union, one assumes that the extrapolated total costs of chronic pain amount to 300 billion euros (Am. Engl.), corresponding to about $1.5-3 \%$ of the GDP (12).

The treatment of back pain, which originates in the spinal region, differs considerably depending on the procedure applied:

Current, evidence-based guidelines on interventional techniques suitable for treating chronic back pain recommend epidural steroid injection in cases of cervical and lumbar disc herniations (13). Furthermore, spinal nerve analgesia and epidural-neuronal injection therapy for treating nerve root compression syndromes constitute a substantial part of conservative therapy (14).

A large randomized study involving over 1,200 patients in the USA who were observed for 4 years, however, demonstrated the superiority of surgical standard laminectomy to conservative therapy (in the study not including epidural steroid injection) for lumbar disc herniations (15).

The absolute indications for discectomy are the paralysis of functionally important muscles or cauda equina syndrome. Severe pain conditions are also considered an indication; however, further specification is lacking. Low back pain only, without radicular symptoms and despite proven disc protrusion, even constitutes a contraindication (16). The range of indications for surgery is thus extremely small and does not include most patients with disc herniations.

Nucleoplasty could possibly close the gap between the various established procedures used to treat back pain. Evidence in favor of nucleoplasty as a relatively new procedure, however, is only described as limited to fair in the current guidelines on interventional techniques for treating chronic back pain (13).

A systematic review by Gerges et al (17) appeared in March 2010 with the title "A Systematic Review on the Effectiveness of the Nucleoplasty Procedure for Discogenic Pain." This review analyzed literature published through September 2008. The investigation mainly focused on the evaluation of pain intensity using visual analog scale / numeric pain scale (VAS/NPS) values. The evaluation of functional capacity in the meta-analysis by Gerges et al (17) is based upon 3 studies, each conducted by the same author (18-20) and using the author's own score. Gerges et al (17) pointed out the insufficient amount of data from the 14 studies-only one of which was randomized-that were included in their meta-analysis. 
In January 2013, a brief systematic review appeared that only included randomized, controlled studies on nucleoplasty applied in patients with disc herniations (21).

Thereafter, a systematic review with the title "An Update of the Systematic Assessment of Mechanical Lumbar Disc Decompression with Nucleoplasty" by Manchikanti et al (22) was published in April 2013, which represents an update of the same authors' article that had appeared in 2009 (23). The review took literature published through September 2012 into consideration. Primary outcome parameters were pain relief and functional improvement, assessed according to the various scores used in the studies chosen for the review. This article, too, concluded that the evidence favoring nucleoplasty was limited to fair, but on no account was it good.

From October 2008 to September 2012, however over a dozen new clinical studies investigating nucleoplasty appeared, many of which again used the VAS value, among others, as an outcome parameter. Manchikanti et al (22) did in fact evaluate these new clinical studies in the context of their systematic review on nucleoplasty; still lacking overall, however, is a metaanalysis supplementing the systematic review that summarizes and interprets the reported results, where possible comprehensively and quantitatively, with the help of statistical methods. Compared to systematic reviews, which are characterized methodically in particular by a detailed study protocol and analysis plan as well as a literature search for suitable studies in accordance with a priori defined inclusion and exclusion criteria, a metaanalysis also provides a quantitative, statistical summary of the results (24).

This paper therefore aimed to improve the scientific evidence on nucleoplasty even further by conducting a supplemental comprehensive meta-analysis. In short, the present meta-analysis deals with the central question of the extent to which it is statistically proven that the nucleoplasty treatment method can positively affect pain relief and functional mobility and lower the complication rate.

The new studies published since October 2008 have made it possible to conduct a meta-analysis of the VAS, a measuring instrument for determining pain intensity (25), and of the Oswestry Disability Index (ODI), a scale that reflects the degree of impairment in terms of percent (26). The present, newly conducted meta-analysis thus quantitatively summarizes VAS and ODI data as well as calculates a total complication rate, which previ- ous systematic reviews were unable to illustrate quantitatively or graphically.

\section{Methods}

In short, it was the intent of the current metaanalysis to answer the question as to the degree to which the nucleoplasty treatment procedure can have a positive effect on pain relief and functional mobility as well as lower the complication rate.

\section{Search Strategy and Selection Criteria}

A systematic search using the terms "nucleoplasty" and/or "plasma disc decompression" was conducted in the MEDLINE database.

Literature published up to and including September 30, 2012, was included.

The inclusion criteria for the meta-analysis were:

1. treatment with nucleoplasty for intervertebral disc conditions

2. publications in English (27)

3. clinical studies with patient populations at defined measurement time points

4. at least 2 or more points on the modified Jadad scale

\section{Outcome parameters to be considered:}

\section{VAS/NPS}

The VAS is a standardized instrument for measuring pain. Patients rate the intensity of their subjectively experienced pain on a $10 \mathrm{~cm}$ scale from 0 (no pain) to 10 (greatest imaginable pain) with a space of one centimeter between the individual values (25).

\section{Complication rate}

The complication rate, specified in percent, describes all adverse events occurring in a patient population.

\section{ODI}

The ODI, developed by Fairbanks et al in 1980, is a 10-item patient-based questionnaire used to make a standardized assessment of functional restriction in spinal mobility caused by back pain (low back pain disability), where minimal impairment is rated with 0 points and maximal impairment 100 points (26).

Complete statistical data sets consisting of sample size, mean, and standard deviation (if appropriate, 
calculation using standard error or the upper and lower quartile) at defined follow-up time points.

\section{Data Collection and Analysis}

Seventy-six articles were identified using the term "nucleoplasty" and 10 publications using "plasma disc decompression." Two papers $(28,29)$ contained both search terms, resulting in a total of 86 studies using both search terms.

Additional literature was found while examining the bibliographies of these 86 articles. It was therefore possible to make a primary evaluation of a total of 125 articles based on their abstracts. We proceeded as follows:

An initial review of the 125 abstracts revealed that 45 articles were not primarily concerned with nucleoplasty, but instead reported on other treatment options for intervertebral disc conditions or only mentioned "nucleoplasty" (30-74).

Therefore, a remaining 80 articles were analyzed. Another 35 of these were excluded because they involved reviews and meta-analyses (18x) (17,23,75-90), basic research (10x) (91-100), guidelines (3x) (101-103), or comments (1x) (104) and not clinical studies with patient case numbers. Also excluded were a case report by Li et al on hemophilia in a patient following nucleoplasty (105), one case report by Zhu et al using nucleoplasty for segment degeneration after fusion surgery (106), and one study by Cuellar et al on failed nucleoplasty (107). All of these articles, upon considering the inclusion criteria, did not address nucleoplasty for intervertebral disc conditions.

At the beginning, we therefore found 45 studies which reported on the clinical application of nucleoplasty. Of these, another 18 studies were excluded for the following reasons:

Even though the patients in the study conducted by Li et al (108) underwent nucleoplasty, the study had to be excluded because the indication was discitis and not disc herniation.

The study by Fabrizi et al (109) could not be included, in which a Coblation-assisted microdiscectomy was performed. Coblation is in fact the underlying technical procedure of nucleoplasty, but it is not usually used in microdiscectomies.

Also, the paper by Theron et al (110) could not be considered since the study addressed the use of "radiopaque gelified ethanol," and nucleoplasty was only applied in addition to this procedure in a subgroup of 11 patients. The study results are therefore not exclu- sively attributable to nucleoplasty.

The articles by Li et al (111), Zakirov et al (112), and Manukovskii et al (113) were published only in Chinese or Russian and were eliminated in accordance with the inclusion criteria. Furthermore, the studies by Cohen et al (114) and Yan et al (115) were not taken into consideration, since they did not specify any precisely defined measurement time points (only mean values of 9 and 29 months, respectively, were mentioned).

The article by Bokov et al (116) described the follow-up period and the patient population of a patient cohort already considered in the meta-analysis (117) and was therefore not included twice.

The 3 case reports by Singh (118), Smuck et al (119), and Puentedura et al (120) as well as the 2 case report series by Pace et al (121) and Chua et al (122) were excluded based on their low level of evidence (123).

The papers by Reverberi et al (124) and Al-Zain et al (125) could not be considered at all for the metaanalysis due to lacking standard deviations for VAS and ODI values, respectively.

To be able to establish the homogeneity essential for a meta-analysis, we set a minimal score of at least 2 points on the modified Jadad scale. Two studies, one by Wolter et al (126) and the other by Bonaldi et al (127), thus dropped out because they only reached a score of one on the modified Jadad scale. The Jadad scale was presented by Jadad et al in Oxford (therefore also called the Oxford scale) in 1996 for making a qualitative assessment of the methodology of studies conducted in pain research. Originally, the Jadad scale consisted of 3 questions pertaining to randomization, blinding, and study dropouts in pain studies (128).

In 2001, Oremus et al added to the original Jadad scale 3 more questions regarding inclusion and exclusion criteria, side effects/negative effects, and statistical methods. Furthermore, one additional point each was given for adequate randomization and blinding, resulting in a total minimal score of 0 and a maximal score of 8 (129).

This meta-analysis implemented the Jadad scale as adapted by Oremus. In order for no systematic error to be made in this meta-analysis, a second, blinded reviewer applied the scale to assess all studies. If the second reviewer reached a different evaluation of a study, a third reviewer was consulted to assess the study in question.

Fig. 1 presents a flowchart of the procedure followed in selecting literature for this meta-analysis and thus in identifying relevant literature for this meta-analysis. 


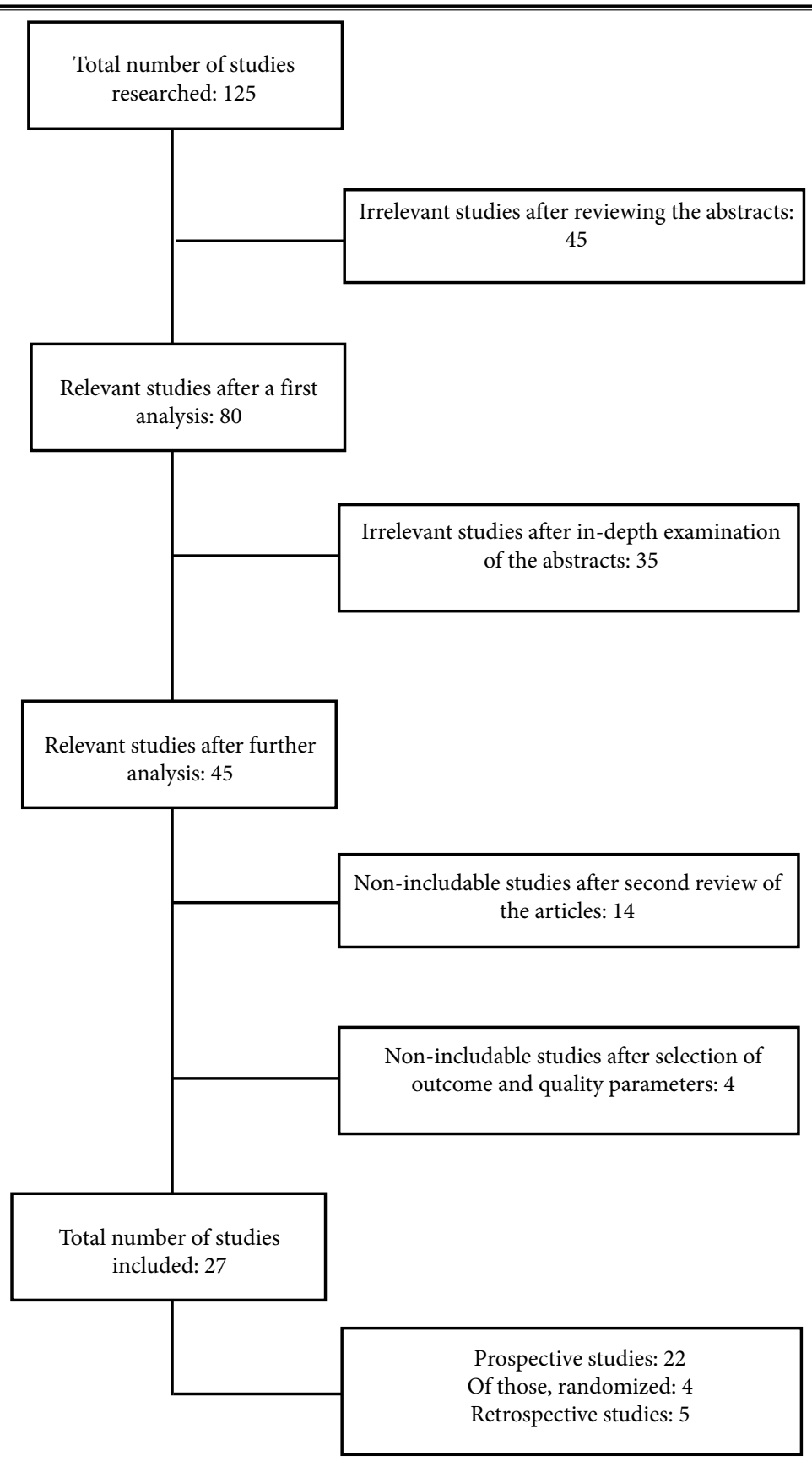

Fig. 1. Flowchart: Identification of relevant studies.

Taking the defined outcome and quality parameters into account, it was possible to include 27 studies this meta-analysis (Table 1).

At first, study values were listed in a table. In the event that no change in the patient number was speci-

fied in the observation period of a study, the number of patients treated at baseline was used for calculation purposes.

Based on the sample sizes and the study values reported in the studies, we performed the statistical calculations using the statistics software called "Com- 
Pain Physician: March/April 2014; 17:E149-E173

Table 1. Salient features of studies included.

\begin{tabular}{|c|c|c|c|c|c|c|c|}
\hline Author & Study design & Follow-up & $\begin{array}{l}\text { Patients treated with } \\
\text { nucleoplasty }\end{array}$ & $\begin{array}{l}\text { VAS/ } \\
\text { NPS } \\
\end{array}$ & $\mathbf{C R}$ & ODI & Segment \\
\hline Alexandre et al (130) & $\begin{array}{l}\text { prospective, non-randomized, } \\
\text { non-controlled }\end{array}$ & 12 months & 1,390 & & $\mathrm{X}$ & & lumbar \\
\hline Azzazi et al (131) & $\begin{array}{l}\text { prospective, non-randomized, } \\
\text { non-controlled }\end{array}$ & 12 months & 50 & $\mathrm{X}$ & $\mathrm{X}$ & $\mathrm{X}$ & lumbar \\
\hline Bhagia et al (132) & $\begin{array}{l}\text { prospective, non-randomized, } \\
\text { non-controlled }\end{array}$ & 2 weeks & 53 & $\mathrm{X}$ & $\mathrm{X}$ & & lumbar \\
\hline Birnbaum (28) & prospective, randomized, controlled & 24 months & $29 / 30$ conservative therapy & $(\mathrm{X})$ & $\mathrm{X}$ & & cervical \\
\hline Bokov et al (117) & $\begin{array}{l}\text { prospective, non-randomized, } \\
\text { controlled }\end{array}$ & 18 months & 73 / 65 microdiscectomy & $\mathrm{X}$ & $\mathrm{X}$ & $\mathrm{X}$ & lumbar \\
\hline Calisaneller et al (133) & $\begin{array}{l}\text { prospective, non-randomized, } \\
\text { non-controlled }\end{array}$ & 6 months & 29 & $\mathrm{X}$ & $\mathrm{X}$ & & lumbar \\
\hline Cesaroni \& Nardi (134) & prospective, randomized, controlled & 12 months & $62 / 53$ conservative therapy & $\mathrm{X}$ & $\mathrm{X}$ & & cervical \\
\hline Cesaroni \& Nardi (135) & $\begin{array}{l}\text { retrospective, non-randomized, } \\
\text { non-controlled }\end{array}$ & 5 years & 349 & & $\mathrm{X}$ & & cervical \\
\hline Gerszten et al (136) & prospective, randomized, controlled & 24 months & $\begin{array}{l}45 \text { / } 40 \text { transforaminal } \\
\text { epidural steroid injection }\end{array}$ & $\mathrm{X}$ & $\mathrm{X}$ & $\mathrm{X}$ & lumbar \\
\hline Gerszten et al (137) & $\begin{array}{l}\text { prospective, non-randomized, } \\
\text { non-controlled }\end{array}$ & 6 months & 67 & $(\mathrm{X})$ & $\mathrm{X}$ & & lumbar \\
\hline Karaman et al (138) & $\begin{array}{l}\text { prospective, non-randomized, } \\
\text { non-controlled }\end{array}$ & 24 months & 56 & $\mathrm{X}$ & $\mathrm{X}$ & $\mathrm{X}$ & lumbar \\
\hline Lemcke et al (139) & $\begin{array}{l}\text { prospective, non-randomized, } \\
\text { controlled }\end{array}$ & 12 months & 96 / 67 Disc Dekompressor & $\mathrm{X}$ & & & lumbar \\
\hline Li et al (140) & $\begin{array}{l}\text { prospective, non-randomized, } \\
\text { non-controlled }\end{array}$ & 12 months & 126 & $\mathrm{X}$ & $\mathrm{X}$ & & cervical \\
\hline Marin (29) & $\begin{array}{l}\text { prospective, non-randomized, } \\
\text { controlled }\end{array}$ & 12 months & $\begin{array}{l}64 / 13 \text { Coblation-assisted } \\
\text { microdiscectomy }\end{array}$ & $(\mathrm{X})$ & $\mathrm{X}$ & & lumbar \\
\hline Masala et al (141) & $\begin{array}{l}\text { prospective, non-randomized, } \\
\text { non-controlled }\end{array}$ & 12 months & 72 & $(\mathrm{X})$ & $\mathrm{X}$ & & lumbar \\
\hline Mirzai et al (142) & $\begin{array}{l}\text { prospective, non-randomized, } \\
\text { non-controlled }\end{array}$ & 12 months & 52 & $\mathrm{X}$ & $\mathrm{X}$ & $\mathrm{X}$ & lumbar \\
\hline Nardi et al (143) & prospective, randomized, controlled & 2 months & $50 / 20$ conservative therapy & $(\mathrm{X})$ & $\mathrm{X}$ & & cervical \\
\hline Reddy et al (144) & $\begin{array}{l}\text { retrospective, non-randomized, } \\
\text { non-controlled }\end{array}$ & 12 months & 49 & $(\mathrm{X})$ & $\mathrm{X}$ & & $\begin{array}{l}\text { lumbar / } \\
\text { thoracic }\end{array}$ \\
\hline Shabat et al (145) & $\begin{array}{l}\text { prospective, non-randomized, } \\
\text { non-controlled }\end{array}$ & 24 months & 87 & $(\mathrm{X})$ & $\mathrm{X}$ & $(\mathrm{X})$ & lumbar \\
\hline Sharps \& Isaac (146) & $\begin{array}{l}\text { prospective, non-randomized, } \\
\text { non-controlled }\end{array}$ & 12 months & 49 & $\mathrm{X}$ & $\mathrm{X}$ & & lumbar \\
\hline Sim et al (147) & $\begin{array}{l}\text { retrospective, non-randomized, } \\
\text { non-controlled }\end{array}$ & 6 months & 22 & $\mathrm{X}$ & $\mathrm{X}$ & & cervical \\
\hline Sinan et al (148) & $\begin{array}{l}\text { prospective, non-randomized, } \\
\text { non-controlled }\end{array}$ & 12 months & 83 & $(\mathrm{X})$ & $\mathrm{X}$ & & lumbar \\
\hline Singh et al (20) & $\begin{array}{l}\text { prospective, non-randomized, } \\
\text { non-controlled }\end{array}$ & 12 months & 47 & $\mathrm{X}$ & $\mathrm{X}$ & & lumbar \\
\hline Singh et al (19) & $\begin{array}{l}\text { prospective, non-randomized, } \\
\text { non-controlled }\end{array}$ & 12 months & 80 & $\mathrm{X}$ & $\mathrm{X}$ & & lumbar \\
\hline Singh et al (18) & $\begin{array}{l}\text { prospective, non-randomized, } \\
\text { non-controlled }\end{array}$ & 12 months & 67 & $\mathrm{X}$ & $\mathrm{X}$ & & lumbar \\
\hline Yakovlev et al (149) & $\begin{array}{l}\text { retrospective, non-randomized, } \\
\text { non-controlled }\end{array}$ & 12 months & 22 & $\mathrm{X}$ & $\mathrm{X}$ & & lumbar \\
\hline Zhu et al (150) & $\begin{array}{l}\text { retrospective, non-randomized, } \\
\text { non-controlled }\end{array}$ & 24 months & 42 & $\mathrm{X}$ & & $\mathrm{X}$ & lumbar \\
\hline
\end{tabular}

$\mathrm{CR}=$ complication rate, $(\mathrm{X})=$ non-includable/non-evaluable study parameters. 
prehensive Meta-Analysis" (Version 2.2.057; 9 December 2010; Biostat, 14 North Dean Street, Englewood, NY, 07631, USA).

The study values for the outcome parameters VAS, complication rate, and ODI were sorted according to measurement time points (for example: baseline, postOP, one week, one month, etc.) and summarized using a random-effects model.

$P$-values for comparison of groups (for example: nucleoplasty versus conservative therapy) were calcu-

Table 2. Systematic presentation of the studies used.

\begin{tabular}{|c|c|c|c|c|c|c|}
\hline Author & $\begin{array}{l}\text { Jada } \\
\text { total }\end{array}$ & $\begin{array}{l}\text { Average } \\
\text { age }\end{array}$ & Age span & $\begin{array}{l}\text { Measurement } \\
\text { time points }\end{array}$ & Inclusion criteria & Exclusion criteria \\
\hline $\begin{array}{l}\text { Alexandre } \\
\text { et al (130) }\end{array}$ & 2 & $\begin{array}{l}\text { not } \\
\text { specified }\end{array}$ & $\begin{array}{l}\text { not } \\
\text { specified }\end{array}$ & $\begin{array}{l}\text { preoperative, } 15 \\
\text { days, } 1,6 \text {, and } \\
12 \text { months after } \\
\text { nucleoplasty with } \\
1,390 \text { patients }\end{array}$ & $\begin{array}{l}\text { chronic lumbar pain with or } \\
\text { without radicular pain lasting } \\
\text { more than } 3 \text { months } \\
\text { absence of neurological } \\
\text { deficit } \\
\text { one level positive provocative } \\
\text { discography }\end{array}$ & $\begin{array}{l}\text { disc herniation with sequestration } \\
\text { large contained herniation that was larger than } \\
\text { one- third the sagittal diameter of the spinal canal } \\
\text { severe spinal stenosis } \\
\text { presence of secondary pain issues } \\
\text { psychological disorders } \\
\text { gait disorders depending on different neurological } \\
\text { or orthopedic pathology }\end{array}$ \\
\hline $\begin{array}{l}\text { Azzazi et al } \\
(131)\end{array}$ & 4 & 41 & $25-61$ & $\begin{array}{l}\text { preoperative, } 2 \\
\text { weeks, } 1,3,6, \\
12 \text { months after } \\
\text { nucleoplasty with } \\
50 \text { patients } \\
\end{array}$ & $\begin{array}{l}\text { disc protrusion or contained } \\
\text { herniated disc }<6 \mathrm{~mm} \text { with } \\
\text { a disc height }>50 \% \text { of the } \\
\text { adjacent disc heights }\end{array}$ & $\begin{array}{l}\text { previous lumbar surgery } \\
\text { significant spinal stenosis } \\
\text { motor weakness } \\
\text { fracture, tumor, spondylolisthesis } \\
\text { more than } 2 \text { symptomatic levels } \\
\end{array}$ \\
\hline $\begin{array}{l}\text { Bhagia et al } \\
(132)\end{array}$ & 4 & 42.1 & $17-78$ & $\begin{array}{l}\text { preoperative, } \\
2 \text { weeks after } \\
\text { nucleoplasty with } \\
53 \text { and } 49 \text { patients }\end{array}$ & $\begin{array}{l}\text { contained disc herniation } \\
\text { with a disc height }>50 \% \text { of } \\
\text { the adjacent disc heights } \\
\text { presence of discogenic axial } \\
\text { back pain or leg pain }\end{array}$ & $\begin{array}{l}\text { sequestered disc } \\
\text { large contained herniation that was larger than } \\
\text { one third the sagittal diameter of the spinal canal } \\
\text { presence of progressive neurologic deficits } \\
\text { spinal instability, fracture, tumor, morbid } \\
\text { obesity, infection } \\
\text { spondylolisthesis } \\
\text { marked spinal stenosis due to extensive } \\
\text { osteophytosis } \\
\text { all patients with axial back pain without } \\
\text { radicular symptoms underwent provocative } \\
\text { discography to confirm concordant pain }\end{array}$ \\
\hline $\begin{array}{l}\text { Birnbaum } \\
(28)\end{array}$ & 3 & $\begin{array}{l}\text { not } \\
\text { specified }\end{array}$ & $23-49$ & $\begin{array}{l}\text { preoperative, } 1 \text { day, } \\
1 \text { week, and } 1,3,6 \text {, } \\
12 \text {, and } 24 \text { months } \\
\text { after nucleoplasty } \\
\text { with } 29,29,29,29, \\
29,29,29 \text {, and } 26 \\
\text { patients }\end{array}$ & $\begin{array}{l}\text { arm pain > back pain } \\
\text { contained disc protrusion or } \\
\text { contained herniated disc not } \\
\text { larger than } 4 \mathrm{~mm} \text { and not } \\
\text { compromising more than } \\
\text { one-fourth of the central } \\
\text { spinal canal }\end{array}$ & $\begin{array}{l}\text { disc height }<50 \% \text { of the adjacent disc heights } \\
\text { evidence of severe disc degeneration } \\
\text { fracture, tumor } \\
\text { moderate/severe spinal stenosis }\end{array}$ \\
\hline $\begin{array}{l}\text { Bokov et al } \\
(117)\end{array}$ & 3 & 43.0 & $\begin{array}{l}\text { not } \\
\text { specified }\end{array}$ & $\begin{array}{l}\text { preoperative, } \\
1,3,6,12 \text {, and } \\
18 \text { months after } \\
\text { nucleoplasty with } \\
73 \text { patients }\end{array}$ & $\begin{array}{l}\text { evidence of nerve root } \\
\text { compression with VAS }>4 \\
\text { and ODI }>40\end{array}$ & $\begin{array}{l}\text { litigation } \\
\text { uncontrolled psychological disorders } \\
\text { evidence of instability of the segment } \\
\text { infection } \\
\text { severe and progressive neurological deficit } \\
\text { previous spinal surgery } \\
\text { spinal stenosis }\end{array}$ \\
\hline $\begin{array}{l}\text { Calisaneller } \\
\text { et al (133) }\end{array}$ & 3 & 44.14 & $32-59$ & $\begin{array}{l}\text { preoperative, } \\
1 \text { day, } 3 \text {, and } 6 \\
\text { months after } \\
\text { nucleoplasty with } \\
29 \text { patients } \\
\end{array}$ & $\begin{array}{l}\text { low back pain and/or leg pain } \\
\text { lasting more than } 6 \text { months } \\
\text { diffuse bulging and/or } \\
\text { protrusion }<5 \mathrm{~mm} \text { at one or } \\
2 \text { levels } \\
\end{array}$ & $\begin{array}{l}\text { disc bulges greater than } 5 \mathrm{~mm} \\
\text { loss of normal disc height greater than } 30 \% \\
\text { previous low-back surgery, neurological deficits } \\
\text { serious medical conditions such as malignancy, } \\
\text { infection or coagulopathy }\end{array}$ \\
\hline $\begin{array}{l}\text { Cesaroni \& } \\
\text { Nardi (134) }\end{array}$ & 6 & 45.03 & $18-75$ & $\begin{array}{l}\text { preoperative }, 6 \\
\text { weeks, } 3,6, \text { and } \\
12 \text { months after } \\
\text { nucleoplasty with } \\
62,62,62,61 \text {, and } \\
61 \text { patients }\end{array}$ & $\begin{array}{l}\text { neck/arm pain VAS }>5 \\
\text { single contained symptomatic } \\
\text { focal disc protrusion between } \\
\text { C3 and T1 } \\
\text { not compromising more than } \\
\text { one-third of the central spinal } \\
\text { canal } \\
\text { minimal corroborative } \\
\text { myotomal deficit } \\
\text { a positive diagnostic nerve } \\
\text { root block } \\
\text { failed to respond to or refused } \\
\text { epidural steroid injection }\end{array}$ & $\begin{array}{l}\text { extruded or sequestered disc larger than } 6 \mathrm{~mm} \\
\text { with a disc height }<50 \% \text { of the adjacent disc } \\
\text { heights } \\
\text { disc prolapse that was larger than one-third the } \\
\text { sagittal diameter of the spinal canal } \\
\text { history of anterior fusion in the cervical level to be } \\
\text { treated } \\
\text { fracture, tumor, infection } \\
\text { central cord lesion in the cervical spine } \\
\text { progressive neurological deficit } \\
\text { hyperostosis causing concurrent foraminal stenosis } \\
\text { at the symptomatic level } \\
\text { myotomal deficit with motor strength less than } 4 / 5 \\
\text { carotid stenosis or significant plaque-like carotid } \\
\text { disease }\end{array}$ \\
\hline
\end{tabular}


Table 2 (cont.). Systematic presentation of the studies used.

\begin{tabular}{|c|c|c|c|c|c|c|}
\hline Author & $\begin{array}{l}\text { Jada } \\
\text { total }\end{array}$ & \begin{tabular}{|l} 
Average \\
age
\end{tabular} & Age span & $\begin{array}{l}\text { Measurement } \\
\text { time points }\end{array}$ & Inclusion criteria & Exclusion criteria \\
\hline $\begin{array}{l}\text { Cesaroni \& } \\
\text { Nardi (135) }\end{array}$ & 2 & \begin{tabular}{|l} 
not \\
specified
\end{tabular} & \begin{tabular}{|l|} 
not \\
specified
\end{tabular} & $\begin{array}{l}\text { preoperative, } 12, \\
24,36,48, \text { and } \\
60 \text { month after } \\
\text { nucleoplasty with } \\
349,302,190 \\
170,124, \text { and } 69 \\
\text { patients }\end{array}$ & $\begin{array}{l}\text { disc protrusion }<3 \mathrm{~mm} \\
\text { not compromising more than } \\
\text { one-fifth of the central spinal } \\
\text { canal }\end{array}$ & $\begin{array}{l}\text { fracture, tumors } \\
\text { acquired stenosis } \\
\text { advanced spondylosis resulting in osseous } \\
\text { foraminal stenosis or disc space collapse } \\
\text { previous spinal surgery } \\
\text { neurological deficit as hypoesthesia or motor } \\
\text { deficits }\end{array}$ \\
\hline $\begin{array}{l}\text { Gerszten et } \\
\text { al (136) }\end{array}$ & 6 & 46 & $20-66$ & $\begin{array}{l}\text { preoperative, } 1, \\
3 \text {, and } 6 \text { months } \\
\text { after nucleoplasty } \\
\text { with } 45,40,30, \\
\text { and } 29 \text { patients } \\
\text { (12 and } 24 \\
\text { months not } \\
\text { reported) }\end{array}$ & $\begin{array}{l}\text { lumbar disc protrusion with } \\
\text { radicular pain score }>5 \text { and } \\
\text { with a disc height }>50 \% \text { of } \\
\text { the adjacent disc heights } \\
\text { BMI less than } 40\end{array}$ & $\begin{array}{l}\text { sciatica originating from more than one disc } \\
\text { level } \\
\text { back pain more than leg pain } \\
\text { cauda equina syndrome } \\
\text { progressive neurological deficit } \\
\text { spondylolisthesis or moderate or severe } \\
\text { stenosis at the level to be treated } \\
\text { previous spinal surgery at or directly adjacent } \\
\text { to the level to be treated } \\
\text { fracture, tumor, infection }\end{array}$ \\
\hline $\begin{array}{l}\text { Gerszten et } \\
\text { al (137) }\end{array}$ & 3 & 41 & $21-70$ & $\begin{array}{l}\text { preoperative, } 3 \text {, } \\
\text { and } 6 \text { months } \\
\text { after nucleoplasty } \\
67,34 \text {, and } 23 \\
\text { patients }\end{array}$ & $\begin{array}{l}\text { leg pain }>\text { back pain } \\
\text { contained disc protrusion } \\
\text { with a disc height }>75 \% \text { of } \\
\text { the adjacent disc heights } \\
\text { discography positive for } \\
\text { concordant pain }\end{array}$ & $\begin{array}{l}\text { complete anular disruption with extruded or } \\
\text { sequestered disc } \\
\text { disc height }<25 \% \text { of adjacent level(s) } \\
\text { moderate to severe spinal stenosis } \\
\text { fracture, tumor, infection }\end{array}$ \\
\hline $\begin{array}{l}\text { Karaman et } \\
\text { al (138) }\end{array}$ & 3 & 40 & $18-59$ & $\begin{array}{l}\text { preoperative, } 1,6, \\
12 \text {, and } 24 \text { months } \\
\text { after nucleoplasty } \\
\text { with } 56,56, \\
54,52 \text {. and } 50 \\
\text { patients }\end{array}$ & $\begin{array}{l}\text { younger than } 60 \text { years } \\
\text { contained single level disc } \\
\text { herniation }<6 \text { mm with } \\
\text { mostly single-side leg pain } \\
\text { with a disc height }>70 \% \text { of } \\
\text { the adjacent disc heights } \\
\text { disc prolapse that was smaller } \\
\text { than one third the sagittal } \\
\text { diameter of the spinal canal }\end{array}$ & $\begin{array}{l}\text { extruded or sequestered disc larger than } 6 \mathrm{~mm} \\
\text { back pain more than leg pain } \\
\text { previous open surgery or percutaneous } \\
\text { intervention on PN planned level } \\
\text { more than one herniation with different levels } \\
\text { instability, tumor, spondylolisthesis } \\
\text { presence of general contraindications, such as } \\
\text { bleeding diathesis, known allergy history for } \\
\text { materials used, psychological disorder, or systemic } \\
\text { infection or local infection in the intervention area }\end{array}$ \\
\hline $\begin{array}{l}\text { Lemcke et } \\
\text { al (139) }\end{array}$ & 3 & 42 & $18-74$ & $\begin{array}{l}\text { preoperative, } \\
\text { postOP/one day, } \\
6 \text {, and } 12 \text { months } \\
\text { after nucleoplasty } \\
\text { with } 96,96,77, \\
\text { and } 69 \text { patients }\end{array}$ & $\begin{array}{l}\text { contained disc herniation } \\
\text { (disc bulging or disc } \\
\text { protrusion) } \\
\text { low back pain and/or } \\
\text { persisting pain radiating to } \\
\text { the lower extremities }\end{array}$ & $\begin{array}{l}\text { extruded or sequestered disc } \\
\text { disc prolapse that was larger than one third the } \\
\text { sagittal diameter of the spinal canal } \\
\text { previously operated segments } \\
\text { severe neurological deficits } \\
\text { co-existing neoplastic or infectious disease }\end{array}$ \\
\hline $\begin{array}{l}\text { Li et al } \\
\text { (140) }\end{array}$ & 3 & 51.9 & $34-66$ & $\begin{array}{l}\text { preoperative, } 2, \\
\text { weeks, } 1,3,6 \text {, and } \\
12 \text { months after } \\
\text { nucleoplasty with } \\
126 \text { patients }\end{array}$ & $\begin{array}{l}\text { contained disc herniation } \\
\text { complaints of radicular pain } \\
\text { with or without neck pain }\end{array}$ & $\begin{array}{l}\text { sequestered herniation } \\
\text { hemorrhagic diasthesis } \\
\text { spondylolisthesis } \\
\text { spinal canal stenosis } \\
\text { ossification of longitudinal ligament (OPLL) } \\
\text { previous surgery at the indicated level } \\
\text { myelopathy }\end{array}$ \\
\hline Marin (29) & 3 & 43 & $23-57$ & $\begin{array}{l}\text { preoperative, } 1 \text {, } \\
3,6,9 \text {, and } 12 \\
\text { months after } \\
\text { nucleoplasty with } \\
64,62,58,47,35 \\
\text { and } 15 \text { patients } \\
\end{array}$ & $\begin{array}{l}\text { back pain with or without } \\
\text { radicular pain } \\
\text { disc protrusion having a disc } \\
\text { height }>30 \% \text { with radicular/ } \\
\text { axial pain }\end{array}$ & $\begin{array}{l}\text { sequestered disc } \\
\text { previous spinal surgery } \\
\text { disc prolapse that was larger than one third the } \\
\text { sagittal diameter of the spinal canal } \\
\text { severe spinal stenosis } \\
\text { progressive neurological deficits }\end{array}$ \\
\hline $\begin{array}{l}\text { Masala et al } \\
(141)\end{array}$ & 4 & 48 & $32-64$ & $\begin{array}{l}\text { preoperative and } \\
12 \text { months after } \\
\text { nucleoplasty with } \\
72 \text { and } 70 \text { patients }\end{array}$ & $\begin{array}{l}\text { lumbalgic and/or sciatalgic } \\
\text { pain due to disc protrusions } \\
\text { and contained herniations } \\
\text { absence of major neurologic } \\
\text { deficit }\end{array}$ & $\begin{array}{l}\text { secondary gain issues, litigation } \\
\text { heavy opioid usage } \\
\text { uncontrolled psychological disorders } \\
\text { extruded or sequestered disc } \\
\text { contained herniation that was larger than one- } \\
\text { third the sagittal diameter of the spinal canal } \\
\text { severe degenerative disk with greater than } 33 \% \\
\text { loss of disk height } \\
\text { non-qualifying results on provocative } \\
\text { discography } \\
\text { marked spinal stenosis due to extensive } \\
\text { osteophytosis } \\
\text { previous spinal surgery in the same region } \\
\text { spondylolisthesis, bone congenital abnormalities } \\
\text { spinal instability, infection, tumor, cauda equina } \\
\text { syndrome }\end{array}$ \\
\hline
\end{tabular}


Table 2 (cont.). Systematic presentation of the studies used.

\begin{tabular}{|c|c|c|c|c|c|c|}
\hline Author & $\begin{array}{l}\text { Jada } \\
\text { total }\end{array}$ & $\begin{array}{l}\text { Average } \\
\text { age }\end{array}$ & Age span & $\begin{array}{l}\text { Measurement } \\
\text { time points }\end{array}$ & Inclusion criteria & Exclusion criteria \\
\hline $\begin{array}{l}\text { Mirzai et al } \\
(142)\end{array}$ & 3 & 44.8 & $\begin{array}{l}\text { not } \\
\text { specified }\end{array}$ & $\begin{array}{l}\text { preoperative, } 2 \text {, } \\
\text { weeks, } 6 \text {, and } \\
12 \text { months after } \\
\text { nucleoplasty with } \\
52,50,50 \text {, and } 47 \\
\text { patients }\end{array}$ & $\begin{array}{l}\text { contained disc herniation } \\
<6 \mathrm{~mm} \text { with a disc height } \\
>50 \% \text { of the adjacent disc } \\
\text { heights } \\
\text { radicular pain }\end{array}$ & $\begin{array}{l}\text { large }(>6 \mathrm{~mm}) \text { or extruded disc herniation } \\
\text { severe degenerative disc material or complete } \\
\text { annular disruption } \\
\text { significant spinal stenosis } \\
\text { older than } 60 \text { years } \\
\text { fracture, tumor, spondylolisthesis } \\
\text { disc height }<50 \% \text {, } \\
\text { back pain greater than leg pain }\end{array}$ \\
\hline $\begin{array}{l}\text { Nardi et al } \\
(143)\end{array}$ & 4 & $\begin{array}{l}\text { not } \\
\text { specified }\end{array}$ & $\begin{array}{l}\text { not } \\
\text { specified }\end{array}$ & $\begin{array}{l}\text { preoperative, } 1 \\
\text { day, } 1 \text { week, and } \\
2 \text { months after } \\
\text { nucleoplasty, with } \\
50 \text { patients }\end{array}$ & $\begin{array}{l}\text { disc protrusion }<3 \mathrm{~mm} \\
\text { not compromising more than } \\
\text { one-fifth of the central spinal } \\
\text { canal } \\
\text { persistent cervical or } \\
\text { unilateral arm pain }\end{array}$ & $\begin{array}{l}\text { acquired stenosis } \\
\text { previous spinal surgery } \\
\text { fracture, tumor } \\
\text { neurological deficit as hypoesthesia or motor } \\
\text { deficits } \\
\text { advanced spondylosis resulting in osseous } \\
\text { foraminal stenosis or disc space collapse }\end{array}$ \\
\hline $\begin{array}{l}\text { Reddy et al } \\
(144)\end{array}$ & 3 & 45 & $22-67$ & $\begin{array}{l}\text { preoperative, } 6, \\
\text { and } 12 \text { months } \\
\text { after nucleoplasty } \\
\text { with } 49 \text { patients }\end{array}$ & $\begin{array}{l}\text { Radicular criteria } \\
\text { leg pain > back pain } \\
\text { contained posterior disc } \\
\text { protrusion } \\
\text { positive discography with } \\
\text { concordant pain or failed } \\
\text { selective nerve root block } \\
\text { Axial criteria } \\
\text { contained central focal disc } \\
\text { protrusion } \\
\text { or positive discography with } \\
\text { concordant pain }\end{array}$ & $\begin{array}{l}\text { a loss of more than } 50 \% \text { of disc height } \\
\text { moderate to severe spinal stenosis } \\
\text { evidence of severe disc degeneration } \\
\text { fracture, tumor }\end{array}$ \\
\hline $\begin{array}{l}\text { Shabat et al } \\
(145)\end{array}$ & 4 & 49 & $22-67$ & $\begin{array}{l}\text { preoperative, } \\
1,3,6,12 \text {, and } \\
24 \text { months after } \\
\text { nucleoplasty with } \\
87,87,87,87,85 \text {, } \\
\text { and } 39 \text { patients }\end{array}$ & $\begin{array}{l}\text { contained disc herniation (up } \\
\text { to } 2 \text { levels) with a disc height } \\
>50 \% \text { of the adjacent disc } \\
\text { heights } \\
\text { radicular low back pain with } \\
\text { or without mechanical low } \\
\text { back pain }\end{array}$ & $\begin{array}{l}\text { sequestered herniation } \\
\text { instability such as spondylolisthesis or } \\
\text { spondylolysis }\end{array}$ \\
\hline $\begin{array}{l}\text { Sharps \& } \\
\text { Isaac (146) }\end{array}$ & 3 & 38 & $30-61$ & $\begin{array}{l}\text { preoperative, } 1,3 \text {, } \\
6 \text {, and } 12 \text { months } \\
\text { after nucleoplasty } \\
\text { with } 49,49, \\
41,24 \text {, and } 13 \\
\text { patients }\end{array}$ & $\begin{array}{l}\text { complaints of back with or } \\
\text { without radicular pain }\end{array}$ & $\begin{array}{l}\text { sequestered herniation } \\
\text { contained herniation that was larger than one- } \\
\text { third the sagittal diameter of the spinal canal } \\
\text { spinal stenosis } \\
\text { presence of progressive neurological deficits } \\
\text { spinal fracture, tumor, infection } \\
\text { participation in any other drug or device study }\end{array}$ \\
\hline $\begin{array}{l}\text { Sim et al } \\
(147)\end{array}$ & 2 & 47.8 & $19-71$ & $\begin{array}{l}\text { preoperative, } 1, \\
\text { and } 6 \text { months } \\
\text { after nucleoplasty } \\
\text { with } 22 \text { patients }\end{array}$ & not specified & not specified \\
\hline $\begin{array}{l}\text { Sinan et al } \\
(148)\end{array}$ & 3 & $\begin{array}{l}\text { not } \\
\text { specified }\end{array}$ & $20-64$ & $\begin{array}{l}\text { preoperative, } 1 \\
\text { week, } 1,3,6,9 \text {, } \\
\text { and } 12 \text { months } \\
\text { after nucleoplasty } \\
\text { with } 83 \text { patients }\end{array}$ & $\begin{array}{l}\text { symptoms of disc protrusion } \\
\text { with a disc height }>50 \% \text { of } \\
\text { the adjacent disc heights }\end{array}$ & $\begin{array}{l}\text { extruded or sequestered disc } \\
\text { previous spinal surgery } \\
\text { severe neurological deficits } \\
\text { instability of the segment, tumor, infection } \\
\text { serious medical conditions }\end{array}$ \\
\hline $\begin{array}{l}\text { Singh et al } \\
(20)\end{array}$ & 4 & 44 & $15-62$ & $\begin{array}{l}\text { preoperative, } 1,3 \text {, } \\
6 \text {, and } 12 \text { months } \\
\text { after nucleoplasty } \\
\text { with } 47,46, \\
42,40 \text {, and } 37 \\
\text { patients }\end{array}$ & \begin{tabular}{|l|} 
discogenic low back pain \\
confirmed by discography \\
with concordant pain VAS $>5$ \\
absence of neurologic deficit
\end{tabular} & $\begin{array}{l}\text { litigation } \\
\text { heavy opioid usage } \\
\text { uncontrolled psychological disorders } \\
\text { disc herniation with sequestration } \\
\text { contained herniation that was larger than one- } \\
\text { third the sagittal diameter of the spinal canal } \\
\text { non-qualifying results on provocative } \\
\text { discography, } \\
\text { spinal instability, infection } \\
\text { marked spinal stenosis due to extensive } \\
\text { osteophytosis }\end{array}$ \\
\hline
\end{tabular}


Table 2 (cont.). Systematic presentation of the studies used.

\begin{tabular}{|c|c|c|c|c|c|c|}
\hline Author & $\begin{array}{l}\text { Jada } \\
\text { total }\end{array}$ & $\begin{array}{l}\text { Average } \\
\text { age }\end{array}$ & Age span & $\begin{array}{l}\text { Measurement } \\
\text { time points }\end{array}$ & Inclusion criteria & Exclusion criteria \\
\hline $\begin{array}{l}\text { Singh et al } \\
\text { (19) }\end{array}$ & 4 & 44.8 & $15-62$ & $\begin{array}{l}\text { preoperative, } 1,3 \text {, } \\
6 \text {, and } 12 \text { months } \\
\text { after nucleoplasty } \\
\text { with } 80,79, \\
75,72 \text {, and } 62 \\
\text { patients }\end{array}$ & $\begin{array}{l}\text { discogenic low back pain } \\
\text { and/or leg pain for } 3 \text { or more } \\
\text { months } \\
\text { confirmed by discography } \\
\text { with concordant pain } \\
\text { absence of neurologic deficit }\end{array}$ & $\begin{array}{l}\text { secondary gain issues } \\
\text { heavy opioid usage } \\
\text { uncontrolled psychological disorders } \\
\text { disc herniation with sequestration } \\
\text { contained herniation that was larger than one } \\
\text { third the sagittal diameter of the spinal canal } \\
\text { infection } \\
\text { marked spinal stenosis due to extensive } \\
\text { osteophytosis } \\
\text { equivocal discography results }\end{array}$ \\
\hline $\begin{array}{l}\text { Singh et al } \\
\text { (18) }\end{array}$ & 4 & 44 & $15-62$ & $\begin{array}{l}\text { preoperative, } 1,3 \text {, } \\
6 \text {, and } 12 \text { months } \\
\text { after nucleoplasty } \\
\text { with } 67,66 \text {, } \\
62,61 \text {, and } 41 \\
\text { patients }\end{array}$ & $\begin{array}{l}\text { contained disc herniation } \\
\text { with presence of discogenic } \\
\text { axial back pain and/or leg } \\
\text { pain } \\
\text { positive provocative } \\
\text { discography with elicitation } \\
\text { of concordant pain and at } \\
\text { least one negative control disc } \\
\text { absence of neurologic deficit }\end{array}$ & $\begin{array}{l}\text { litigation } \\
\text { heavy opioid usage } \\
\text { disc herniation with sequestration } \\
\text { contained herniation that was larger than one- } \\
\text { third the sagittal diameter of the spinal canal } \\
\text { uncontrolled psychological disorders } \\
\text { non-qualifying results on provocative } \\
\text { discography } \\
\text { spinal instability, infection } \\
\text { marked spinal stenosis due to extensive } \\
\text { osteophytosis }\end{array}$ \\
\hline $\begin{array}{l}\text { Yakovlev et } \\
\text { al (149) }\end{array}$ & 4 & 39 & $22-51$ & $\begin{array}{l}\text { preoperative, } 1,3 \text {, } \\
6 \text {, and } 12 \text { months } \\
\text { after nucleoplasty } \\
\text { with } 22 \text { patients }\end{array}$ & $\begin{array}{l}\text { contained disc protrusion } \\
\text { with a disc height }>50 \% \text { of } \\
\text { the adjacent disc heights } \\
\text { discography confirming } \\
\text { concordant pain at each } \\
\text { suspected level and ruling out } \\
\text { involvement at other levels } \\
\text { radicular or axial low back } \\
\text { pain of } 6 \text { or more months } \\
\text { absence of neurologic deficit }\end{array}$ & $\begin{array}{l}\text { disc sequestration } \\
\text { spinal stenosis } \\
\text { more than } 2 \text { symptomatic levels } \\
\text { history of open disk surgery at suspected levels } \\
\text { fracture, infection, tumor } \\
\text { prominent coexisting psychological disorders }\end{array}$ \\
\hline $\begin{array}{l}\text { Zhu et al } \\
\text { (150) }\end{array}$ & 2 & 39.8 & $21-56$ & $\begin{array}{l}\text { preoperative, } 1 \\
\text { week, } 12 \text {, and } \\
24 \text { months after } \\
\text { nucleoplasty with } \\
42 \text { patients }\end{array}$ & $\begin{array}{l}\text { contained disc protrusion } \\
\text { with a disc height }>50 \% \text { of } \\
\text { the adjacent disc heights } \\
\text { discogenic low back pain } \\
\text { discography confirming } \\
\text { concordant pain at each } \\
\text { suspected level }\end{array}$ & $\begin{array}{l}\text { disc protrusion that was larger than one-third } \\
\text { the sagittal diameter of the spinal canal } \\
\text { spinal stenosis } \\
\text { previously operated segments } \\
\text { severe neurological deficits } \\
\text { spinal tumors } \\
\text { infectious diseases }\end{array}$ \\
\hline
\end{tabular}

Table 3. Systematic presentation of perioperative data from the studies used.

\begin{tabular}{|c|c|c|c|c|c|c|}
\hline Author & \begin{tabular}{|l|} 
Pre- \\
operative \\
diagnostics
\end{tabular} & Prior treatment & $\begin{array}{l}\text { Perioperative } \\
\text { antibiotics }\end{array}$ & Anesthesia & $\begin{array}{l}\text { Additional } \\
\text { treatment } \\
\text { directly after } \\
\text { nucleoplasty }\end{array}$ & $\begin{array}{l}\text { Additional outcome } \\
\text { parameters }\end{array}$ \\
\hline $\begin{array}{l}\text { Alexandre et } \\
\text { al (130) }\end{array}$ & MRI/CT & $\begin{array}{l}3 \text { months of conservative } \\
\text { therapy }\end{array}$ & none & $\begin{array}{l}\text { intravenous } \\
\text { sedation }\end{array}$ & none & $\begin{array}{l}\text { MRI/CT, JOA Score } \\
\text { Scale }\end{array}$ \\
\hline $\begin{array}{l}\text { Azzazi et al } \\
(131)\end{array}$ & MRI & $\begin{array}{l}3 \text { months of conservative } \\
\text { therapy }\end{array}$ & $\begin{array}{l}\text { prophylactic } \\
\text { intravenous } \\
\text { antibiotic }\end{array}$ & $\begin{array}{l}\text { local anesthesia } \\
\text { or monitored } \\
\text { anesthesia }\end{array}$ & none & $\begin{array}{l}\text { Analgesic } \\
\text { consumption, MRI/CT }\end{array}$ \\
\hline $\begin{array}{l}\text { Bhagia et al } \\
(132)\end{array}$ & not specified & $\begin{array}{l}6 \text { weeks of conservative } \\
\text { therapy including epidural } \\
\text { steroid injections for axial } \\
\text { pain, selective nerve root } \\
\text { injections for radicular pain }\end{array}$ & none & not specified & $\begin{array}{l}2.0 \mathrm{~mL} \\
\text { betamethasone } \\
\text { or Depo-Medrol } \\
\text { and } 1.0 \mathrm{~mL} \text { of } \\
1.0 \% \text { Xylocaine } \\
\text { for radicular } \\
\text { symptoms }\end{array}$ & not specified \\
\hline $\begin{array}{l}\text { Birnbaum } \\
(28)\end{array}$ & MRI & $\begin{array}{l}2-3 \text { months of } \\
\text { conservative therapy } \\
\text { including epidural steroid } \\
\text { injections and selective } \\
\text { nerve root blocks }\end{array}$ & $2 \mathrm{~g}$ cefazolin & $\begin{array}{l}\text { local anesthesia } \\
\text { and under } \\
\text { analgosedatives }\end{array}$ & none & not specified \\
\hline $\begin{array}{l}\text { Bokov et al } \\
\text { (117) }\end{array}$ & $\begin{array}{l}\text { MRI, } \\
\text { optional CT }\end{array}$ & $\begin{array}{l}\text { conservative therapy } \\
\text { including selective nerve } \\
\text { root blocks }\end{array}$ & none & $\begin{array}{l}\text { intravenous } \\
\text { sedation }\end{array}$ & $\begin{array}{l}\text { betamethasone } \\
\text { and lidocaine }\end{array}$ & not specified \\
\hline
\end{tabular}


Nucleoplasty, a Minimally Invasive Procedure for Disc Decompression

Table 3 (cont.). Systematic presentation of perioperative data from the studies used.

\begin{tabular}{|c|c|c|c|c|c|c|}
\hline Author & \begin{tabular}{|l|} 
Pre- \\
operative \\
diagnostics
\end{tabular} & Prior treatment & $\begin{array}{l}\text { Perioperative } \\
\text { antibiotics }\end{array}$ & Anesthesia & \begin{tabular}{|l|} 
Additional \\
treatment \\
directly after \\
nucleoplasty
\end{tabular} & $\begin{array}{l}\text { Additional outcome } \\
\text { parameters }\end{array}$ \\
\hline $\begin{array}{l}\text { Calisaneller et } \\
\text { al (133) }\end{array}$ & MRI & $\begin{array}{l}6 \text { weeks of conservative } \\
\text { therapy }\end{array}$ & none & local anesthesia & none & MRI/CT \\
\hline $\begin{array}{l}\text { Cesaroni \& } \\
\text { Nardi (134) }\end{array}$ & MRI & $\begin{array}{l}30 \text { days of conservative } \\
\text { therapy }\end{array}$ & cephalosporin & $\begin{array}{l}\text { intravenous } \\
\text { sedation }\end{array}$ & none & not specified \\
\hline $\begin{array}{l}\text { Cesaroni \& } \\
\text { Nardi }(135)\end{array}$ & not specified & conservative therapy & cephalosporin & $\begin{array}{l}\text { intravenous } \\
\text { sedation }\end{array}$ & none & $\begin{array}{l}\text { SF } 36 \text { (36-Item Short } \\
\text { Form Health Survey), } \\
\text { NDI (Neck Disability } \\
\text { Index) }\end{array}$ \\
\hline $\begin{array}{l}\text { Gerszten et al } \\
\text { (136) }\end{array}$ & not specified & epidural steroid injections & none & not specified & none & $\begin{array}{l}\text { satisfaction with } \\
\text { treatment, SF } 36 \\
\text { (36-Item Short Form } \\
\text { Health Survey) }\end{array}$ \\
\hline $\begin{array}{l}\text { Gerszten et al } \\
\text { (137) }\end{array}$ & MRI & $\begin{array}{l}6 \text { weeks of conservative } \\
\text { therapy }\end{array}$ & none & $\begin{array}{l}\text { local anesthetic } \\
\text { or induction of } \\
\text { general anesthesia }\end{array}$ & none & $\begin{array}{l}\text { SF 36 (36-Item Short } \\
\text { Form Health Survey), } \\
\text { EQ 5D (EuroQol 5D) }\end{array}$ \\
\hline $\begin{array}{l}\text { Karaman et al } \\
(138)\end{array}$ & MRI & $\begin{array}{l}\text { conservative therapy at } \\
\text { least for } 6 \text { weeks within the } \\
\text { last } 6 \text { months }\end{array}$ & $1 \mathrm{~g}$ cefazolin & local anesthesia & none & $\begin{array}{l}\text { satisfaction with } \\
\text { treatment }\end{array}$ \\
\hline $\begin{array}{l}\text { Lemcke et al } \\
\text { (139) }\end{array}$ & MRI & $\begin{array}{l}6 \text { weeks of conservative } \\
\text { therapy }\end{array}$ & $1.5 \mathrm{~g}$ cefazolin & local anesthesia & none & $\begin{array}{l}\text { analgesic } \\
\text { consumption, ability } \\
\text { to work, disability in } \\
\text { daily life }\end{array}$ \\
\hline Li et al (140) & MRI and CT & $\begin{array}{l}6 \text { weeks of conservative } \\
\text { therapy }\end{array}$ & not clear defined & local anesthesia & none & $\begin{array}{l}\text { Macnab criteria, } \\
\text { segment stability }\end{array}$ \\
\hline Marin (29) & MRI & $\begin{array}{l}6 \text { weeks of conservative } \\
\text { therapy }\end{array}$ & $1 \mathrm{~g}$ cefazolin & $\begin{array}{l}\text { local anesthesia } \\
\text { and intravenous } \\
\text { sedation }\end{array}$ & none & $\begin{array}{l}\text { analgesic } \\
\text { consumption, } \\
\text { satisfaction with } \\
\text { treatment, return to } \\
\text { work }\end{array}$ \\
\hline $\begin{array}{l}\text { Masala et al } \\
(141)\end{array}$ & MRI & $\begin{array}{l}6 \text { weeks of conservative } \\
\text { therapy }\end{array}$ & none & $\begin{array}{l}\text { intravenous } \\
\text { sedation }\end{array}$ & none & $\begin{array}{l}\text { satisfaction with } \\
\text { treatment, MRI/CT }\end{array}$ \\
\hline $\begin{array}{l}\text { Mirzai et al } \\
(142)\end{array}$ & MRI & $\begin{array}{l}3 \text { months of conservative } \\
\text { therapy }\end{array}$ & none & local anesthesia & none & $\begin{array}{l}\text { analgesic } \\
\text { consumption, } \\
\text { satisfaction with } \\
\text { treatment }\end{array}$ \\
\hline $\begin{array}{l}\text { Nardi et al } \\
(143)\end{array}$ & MRI & conservative therapy & cephalosporin & $\begin{array}{l}\text { intravenous } \\
\text { sedation }\end{array}$ & none & $\mathrm{MRI} / \mathrm{CT}$ \\
\hline $\begin{array}{l}\text { Reddy et al } \\
(144)\end{array}$ & MRI & $\begin{array}{l}3 \text { months of conservative } \\
\text { therapy }\end{array}$ & $1 \mathrm{~g}$ cefazolin & $\begin{array}{l}\text { local anesthesia } \\
\text { and intravenous } \\
\text { sedation }\end{array}$ & $\begin{array}{l}5 \mathrm{cc}(=\mathrm{mL}) 0.25 \% \\
\text { bupivacaine } \\
\text { und } 60 \mathrm{mg} \\
\text { methylprednisolone }\end{array}$ & $\begin{array}{l}\text { analgesic } \\
\text { consumption, } \\
\text { satisfaction with } \\
\text { treatment, work \& } \\
\text { leisure impairment }\end{array}$ \\
\hline $\begin{array}{l}\text { Shabat et al } \\
\text { (145) }\end{array}$ & MRI/CT & $\begin{array}{l}6 \text { months of conservative } \\
\text { therapy including epidural } \\
\text { steroid injections }\end{array}$ & $1 \mathrm{~g}$ Cefamyzin i.v. & local anesthesia & none & not specified \\
\hline $\begin{array}{l}\text { Sharps \& } \\
\text { Isaac (146) }\end{array}$ & not specified & $\begin{array}{l}6 \text { weeks of conservative } \\
\text { therapy (including epidural } \\
\text { steroid injections for } \\
\text { radicular symptoms) }\end{array}$ & $\begin{array}{l}1 \text { gm of intravenous } \\
\text { cefazolin and } \\
500 \text { mg of oral } \\
\text { Cephalexin every } \\
6 \text { hours for } 48 \\
\text { hours. Patients } \\
\text { with penicillin } \\
\text { or cephalosporin } \\
\text { allergy: } 400 \mathrm{mg} \\
\text { of intravenous } \\
\text { ciprofloxacin prior } \\
\text { to the procedure and } \\
500 \text { mg orally twice } \\
\text { a day for } 48 \text { hours. }\end{array}$ & local anesthesia & none & $\begin{array}{l}\text { analgesic } \\
\text { consumption, } \\
\text { satisfaction with } \\
\text { treatment, return to } \\
\text { work }\end{array}$ \\
\hline
\end{tabular}


Table 3 (cont.). Systematic presentation of perioperative data from the studies used.

\begin{tabular}{|c|c|c|c|c|c|c|}
\hline Author & \begin{tabular}{|l} 
Pre- \\
operative \\
diagnostics
\end{tabular} & Prior treatment & $\begin{array}{l}\text { Perioperative } \\
\text { antibiotics }\end{array}$ & Anesthesia & $\begin{array}{l}\text { Additional } \\
\text { treatment } \\
\text { directly after } \\
\text { nucleoplasty }\end{array}$ & $\begin{array}{l}\text { Additional outcome } \\
\text { parameters }\end{array}$ \\
\hline $\begin{array}{l}\text { Sim et al } \\
(147)\end{array}$ & MRI & not specified & $1 \mathrm{~g}$ cefazolin & $\begin{array}{l}\text { local anesthesia } \\
\text { and on-demand } \\
\text { intravenous } \\
\text { sedation } \\
\end{array}$ & none & Macnab criteria \\
\hline $\begin{array}{l}\text { Sinan et al } \\
(148)\end{array}$ & MRI & $\begin{array}{l}2 \text { weeks of conservative } \\
\text { therapy partially including } \\
\text { epidural steroid injections }\end{array}$ & none & $\begin{array}{l}\text { intravenous } \\
\text { sedation }\end{array}$ & none & RMDQ \\
\hline $\begin{array}{l}\text { Singh et al } \\
(20)\end{array}$ & not specified & $\begin{array}{l}3 \text { months or more of } \\
\text { conservative therapy } \\
\text { including injection therapy }\end{array}$ & $\begin{array}{l}\text { intradiscal or } \\
\text { intravenous } \\
\text { antibiotics }\end{array}$ & $\begin{array}{l}\text { intravenous } \\
\text { sedation }\end{array}$ & none & $\begin{array}{l}\text { functional } \\
\text { improvement }\end{array}$ \\
\hline $\begin{array}{l}\text { Singh et al } \\
\text { (19) }\end{array}$ & not specified & $\begin{array}{l}3 \text { months or more of } \\
\text { conservative therapy } \\
\text { including injection therapy }\end{array}$ & none & $\begin{array}{l}\text { monitored } \\
\text { anesthesia }\end{array}$ & none & $\begin{array}{l}\text { functional } \\
\text { improvement }\end{array}$ \\
\hline $\begin{array}{l}\text { Singh et al } \\
\text { (18) }\end{array}$ & not specified & $\begin{array}{l}3 \text { months or more of } \\
\text { conservative therapy } \\
\text { including injection therapy }\end{array}$ & none & $\begin{array}{l}\text { local anesthesia } \\
\text { and monitored } \\
\text { anesthesia }\end{array}$ & none & $\begin{array}{l}\text { functional } \\
\text { improvement }\end{array}$ \\
\hline $\begin{array}{l}\text { Yakovlev et al } \\
\text { (149) }\end{array}$ & MRI & $\begin{array}{l}\text { conservative therapy } \\
\text { including epidural steroid } \\
\text { injections and selective } \\
\text { nerve root injections }\end{array}$ & $\begin{array}{l}\text { 40mg cefazolin } \\
\text { (local) }\end{array}$ & $\begin{array}{l}\text { monitored } \\
\text { anesthesia }\end{array}$ & $\begin{array}{l}2 \mathrm{~mL} 0.25 \% \\
\text { bupivacaine }\end{array}$ & $\begin{array}{l}\text { analgesic } \\
\text { consumption, return } \\
\text { to work, functional } \\
\text { status }\end{array}$ \\
\hline $\begin{array}{l}\text { Zhu et al } \\
(150)\end{array}$ & MRI & $\begin{array}{l}6 \text { months of conservative } \\
\text { therapy }\end{array}$ & $1.5 \mathrm{~g}$ cefazolin & local anesthesia & $\begin{array}{l}\text { For patients } \\
\text { with radicular } \\
\text { symptoms, } 2.0 \mathrm{~mL} \\
\text { of betamethasone } \\
\text { and } 1.0 \mathrm{~mL} \text { of } \\
1.0 \% \text { lidocaine }\end{array}$ & not specified \\
\hline
\end{tabular}

lated with Z-Statistik software.

The 27 studies we used were compiled in 2 tables in the context of this systematic review (Tables 2 and 3 ).

\section{VAS}

If in a study several numerical VAS/NPS figures were found because various pain intensities had been documented for various body regions of the patients, the arithmetic mean was calculated to harmonize the VAS/NPS data for "back pain" and "leg/radicular pain."

This step was necessary to calculate a total value for one measurement time point defined in the respective studies by Bhagia et al (132), Gerszten et al (136), Lemcke et al (139), and Zhu et al (150).

The VAS values for "numbness" from the study by Zhu et al (150) were the only values not included in the calculation, since they clearly did not involve pain assessments.

The paper by Bhagia et al (132) was taken into consideration. Although no standard deviation was indicated for the defined measurement time point, the standard deviation of the change in VAS was indeed specified. This standard deviation was able to be applied for the baseline value assuming a correlation of
0.5 between the baseline and the 2-week values. This decision was based on the "Cochrane Handbook for Systematic Reviews for Interventions" (151).

Furthermore, it was possible to include the article by Singh et al (19) published in 2003 in the meta-analysis. The missing standard deviation for the baseline value was able to be taken from the article by Singh et al (18) from 2002 in accordance with the "Cochrane Handbook for Systematic Reviews for Interventions" (151); in general, both studies reported strikingly similar values.

The data reported by Bokov et al (117) in the nucleoplasty group had to be calculated separately, because in this study 3 differently sized subgroups having patients with differently sized disc herniations had undergone nucleoplasty. Based on the respective group size, we were able to calculate the proportion of single values with respect to the new total value.

Due to missing standard deviations for VAS values, we could not include the paper by Birnbaum (28) in the VAS calculation. The same applies to the articles by Gerszten et al (137), Masala et al (141), Shabat et al (145), and Sinan et al (148). 
The papers by Marin (29), Nardi et al. (143), and Reddy et al (144) did not even report study values for the most part, and they only described an evaluation of the VAS.

These studies are therefore listed in Table 1 in parentheses. They were still included in the metaanalysis, however, because they contained data on other outcome parameters considered in the current meta-analysis.

It was possible to generate a control group called "conservative therapy" (including epidural steroid injection) from the control groups of the 27 studies in Table 1 that were included for this meta-analysis.

We decided to assign patients treated with epidural steroid injection to the control group "conservative therapy" because other studies proceeded likewise $(152,153)$.

Here too, however, the studies by Nardi et al (143) and Birnbaum (28) could not be taken into consideration due to missing study data or standard deviations, as was the case for the analysis of VAS data on nucleoplasty.

\section{Complication Rate}

The complication rate calculated here includes all events assessed in the studies as complications. Most of the studies reported no or no significant complications. If no information on complications was found, the study had to be excluded from the calculation of the meta-analysis.

However, if it was reported that no complications had occurred and clinically relevant problems after nu- cleoplasty were still described, these values were then adjusted correspondingly based on the description for this meta-analysis. This had to be followed for the papers by Bhagia et al from 2006 (132) and Sinan et al from 2011 (148) described below.

\section{ODI}

The ODI values for the nucleoplasty group in the study by Bokov et al (117) had to be calculated as the ones for VAS/NPS. Based on the respective group size and the study values, it was possible to calculate a new total value from 3 differently sized subgroups treated with nucleoplasty for disc herniations of different severities.

The study by Shabat et al (145) reported neither exact ODI values nor standard errors; therefore, we did not take this article into account in the meta-analysis.

\section{Results}

The remaining 27 articles consisted of 22 prospective and 5 retrospective studies. Among these 27 clinical studies, 4 were randomized, controlled studies and 3 others were controlled, non-randomized studies. The remaining 20 studies were non-interventional studies (NIS). Altogether, 3,211 patients were treated with nucleoplasty in the 27 studies.

\section{VAS}

In the 17 studies using VAS/NPS as outcome parameters, 971 patients underwent nucleoplasty. Four of these studies had control groups comprising 230 patients total. Nucleoplasty affected a significant drop

Table 4. Pain reduction after nucleoplasty total (cervical + lumbar).

\begin{tabular}{|c|c|c|c|c||}
\hline Random effects analysis & Patients & $\begin{array}{c}\text { VAS / NPS } \\
\text { nucleoplasty total }\end{array}$ & 95\% CI & P values in comparison to baseline \\
\hline Baseline & 971 & 7.27 & {$[7.03 ; 7.51]$} & $<0.001$ \\
\hline 1 day & 125 & 2.12 & {$[1.65 ; 2.59]$} & $<0.001$ \\
\hline 1 week & 42 & 2.50 & {$[2.42 ; 2.58]$} & $<0.001$ \\
\hline 2 weeks & 275 & 2.70 & {$[1.72 ; 3.68]$} & $<0.001$ \\
\hline 1 month & 589 & 3.23 & {$[2.57 ; 3.89]$} & $<0.001$ \\
\hline 6 weeks & 102 & 2.66 & {$[2.59 ; 2.73]$} & $<0.001$ \\
\hline 3 months & 612 & 2.84 & {$[2.45 ; 3.23]$} & $<0.001$ \\
\hline 6 months & 790 & 3.06 & {$[2.60 ; 3.53]$} & $<0.001$ \\
\hline 12 months & 702 & 3.03 & {$[1.15 ; 3.92]$} & $<0.001$ \\
\hline 18 months & 73 & 1.54 & {$[3.34 ; 4.04]$} & $<0.001$ \\
\hline 24 months & 92 & 3.69 & & \\
\hline
\end{tabular}


Pain Physician: March/April 2014; 17:E149-E173

Table 5. Pain reduction after conservative therapy (including epidural steroid injection).

\begin{tabular}{|c|c|c|c|c|}
\hline $\begin{array}{c}\text { Random effects } \\
\text { analysis }\end{array}$ & Patients & $\begin{array}{c}\text { VAS / NPS } \\
\text { conservative therapy }\end{array}$ & 95\% CI & $\begin{array}{c}\text { P values in comparison } \\
\text { to nucleoplasty total }\end{array}$ \\
\hline Baseline & 98 & 6.98 & {$[5.91 ; 8.04]$} & 0.599 \\
\hline 6 weeks & 91 & 5.76 & {$[5.20 ; 6.33]$} & $*<0.001$ \\
\hline 3 months & 88 & 4.87 & {$[3.86 ; 5.89]$} & $*<0.001$ \\
\hline 6 months & 85 & 4.25 & {$[2.61 ; 5.90]$} & 0.173 \\
\hline 12 months & 57 & 3.85 & {$[3.77 ; 3.92]$} & 0.073 \\
\hline
\end{tabular}

* Significant improvement of nucleoplasty in comparison to conservative therapy $(P<0.05)$

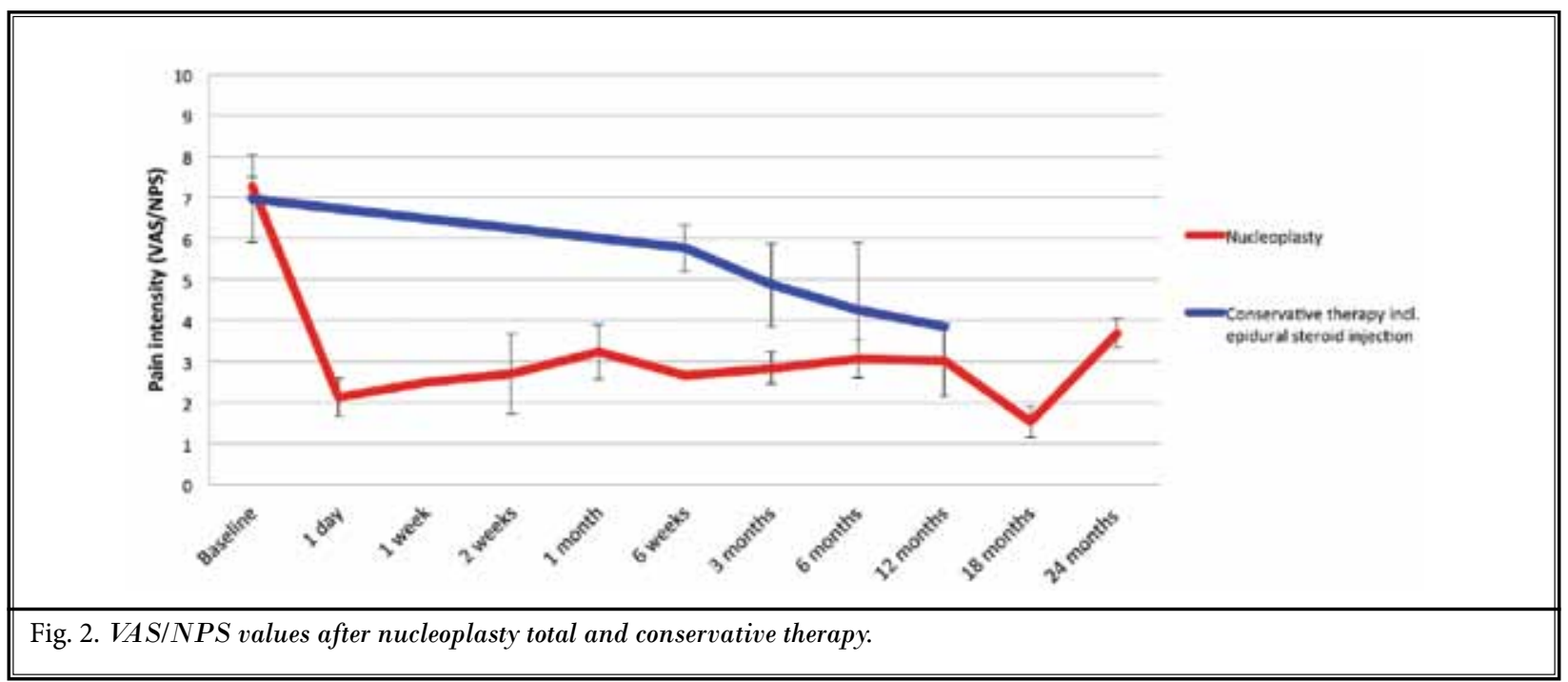

in pain intensity compared to baseline at every measurement time point (Table 4).

In contrast, conservative therapy did not show a significant difference compared to baseline until after one year. Nucleoplasty was significantly superior to conservative therapy after 6 weeks and after 3 months (Table 5).

Fig. 2 shows the nucleoplasty VAS/NPS values calculated in the meta-analysis compared to conservative therapy (including epidural steroid injection), depicted at each of the measurement time points in the included studies.

The calculated VAS/NPS values for cervical nucleoplasty as well as those for lumbar nucleoplasty are presented in Fig. 3, likewise depicted at the measurement time points in the included studies. In all, 761 patients with lumbar and 210 patients with cervical disc herniations were treated. Cervical nucleoplasty appeared to bring greater pain relief compared to lumbar nucleoplasty; the difference, however, was not significant at any time point.

The values for the subgroup analysis for microdiscectomy and Dekompressor were not depicted because comparative individual studies have illustrated them sufficiently $(29,117,139)$. These values, however, were taken into consideration when calculating the nucleoplasty VAS/NPS values.

\section{Complication Rate}

Twenty-five studies comprising 3,069 patients in the nucleoplasty group were available for calculating the complication rate. Five studies had a control group with 168 patients total. The complication rate was $1.5 \%$ for nucleoplasty and $4.0 \%$ for the entire group of control procedures (Figs. 4 and 5). The most frequent complications were postoperative discitis and tingling/ numbness or leg pain.

Since this value for nucleoplasty involves a pooled value from a meta-analysis, the true value for nucleoplasty lies between $0.7 \%$ and $3.0 \%$, thus definitely in 


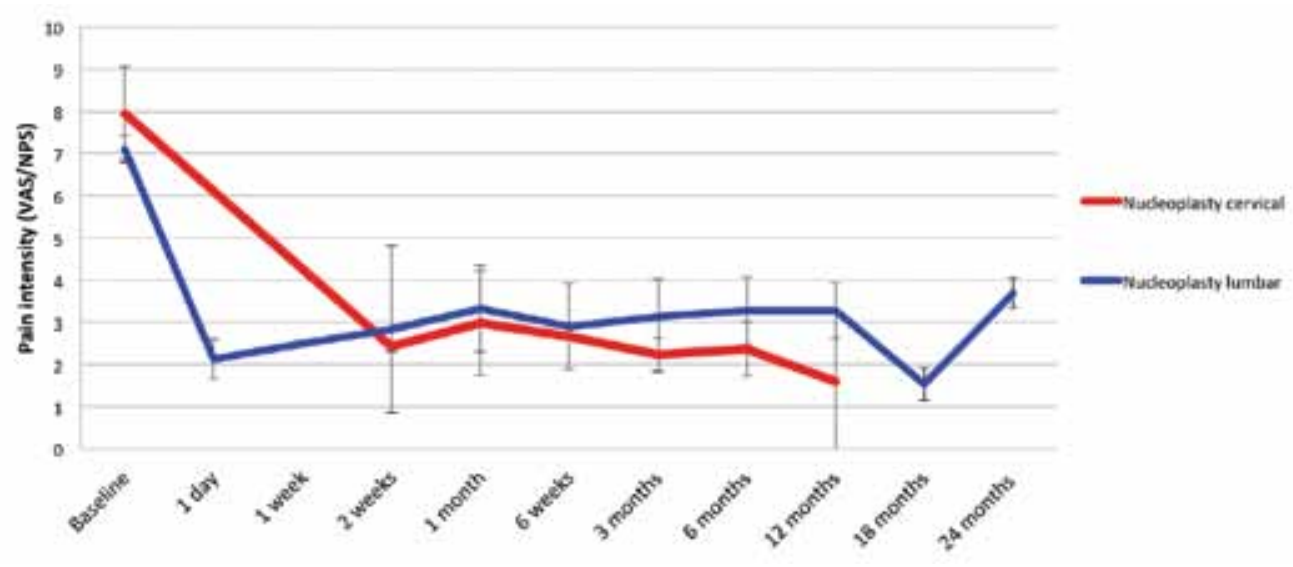

Fig. 3. VAS/NPS values after nucleoplasty for herniated cervical and lumbar discs.

\section{Complication rate Nucleoplasty}

Study name

Azzazi 2011-9

Alexandre 2005-1

Bhagia 2006-1

Birnbaum 2009-6

Bokov 2010-9

Calisaneller 2007-4

Cesaroni 2010-3

Cesaroni 2011-8

Gerszten 2006-1

Gerszten 2010-4

Karaman 2011-8

Li 2008-12

Marin 2005-1

Masala 2007-5

Mirzai 2007-1

Nardi 2005-1

Reddy 2005-10

Shabat 2012-8

Sharps 2002-4

Sim 2011-3

Sinan 2011-8

Singh 2002-7

Singh 2003-7

Singh 2004-10

Yakovlev 2007-3
Statistics for each study

Lower

limit

0,000

0,113

0,001

0,000

0,001

0,000

0,000

0,000

0,047

0,001

0,001

0,000

0,000

0,001

0,001

0,001

0,000

0,001

0,001

0,012

0,000

0,000

0,001

0,001

0,007

$\begin{gathered}\text { Upper } \\ \text { limit }\end{gathered}$
0,219
0,006
0,339
0,217
0,099
0,217
0,115
0,020
0,107
0,241
0,125
0,054
0,111
0,100
0,134
0,138
0,141
0,084
0,141
0,268
0,106
0,107
0,091
0,146
0,268
0,030

Event rate and $95 \% \mathrm{Cl}$

Total

5 / 50

$0 / 1390$

$10 / 49$

$0 / 29$

$0 / 73$

$0 / 29$

$0 / 62$

$1 / 349$

$0 / 67$

$5 / 45$

$0 / 56$

$1 / 126$

$0 / 64$

$0 / 72$

$0 / 52$

$0 / 50$

$0 / 49$

$0 / 87$

$0 / 49$

$0 / 22$

$3 / 83$

$0 / 67$

$0 / 80$

$0 / 47$

$0 / 22$

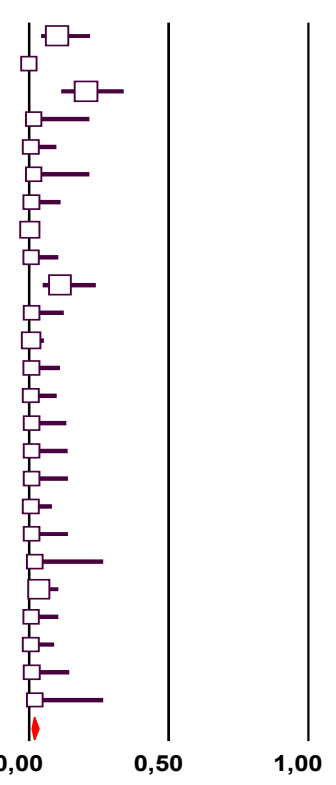

\section{Meta Analysis}

Fig. 4. Meta-analysis complication rate-nucleoplasty. 


\section{Complication rate}

\section{Study name}

Ever

Birnbaum 2009-6

Bokov 2010-9

Gerszten 2010-4

Marin 2005-1

Nardi 2005-1

\section{Statistics for each study}

\section{Event}

rate

0,016

0,008

0,175

0,036

0,024

0,040

$\begin{gathered}\text { Lower } \\ \text { limit }\end{gathered}$
0,001
0,000
0,086
0,002
0,001
0,009

Upper limit

0,211

0,110

0,324

0,384

0,287

0,162

\section{Event rate and $95 \% \mathrm{Cl}$}

Total

$0 / 30$

$0 / 65$

$7 / 40$

$0 / 13$

$0 / 20$

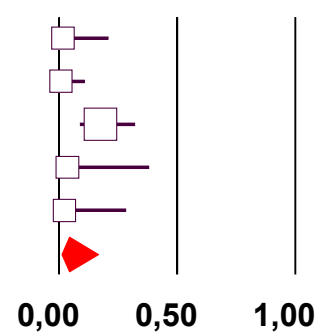

Fig. 5. Meta-analysis complication rate-control group procedures.

\section{Complication rate Nucleoplasty cervical}

\section{Study name}

Birnbaum 2009-6

Cesaroni 2010-3

Cesaroni 2011-8

Li 2008-12

Nardi 2005-1

Sim 2011-3

\section{Event
rate}

\begin{abstract}
0,017
\end{abstract}
0,008

0,003

0,008

0,010

0,022

0,008

\section{Statistics for each study}

$\begin{array}{cc}\text { Lower } & \text { Upper } \\ \text { limit } & \text { limit }\end{array}$

\begin{abstract}
0,001
\end{abstract}
0,000

0,000

0,001

0,001

0,001

0,003
0,217

0,115

0,020

0,054

0,138

0,268

0,021
Event rate and $95 \% \mathrm{CI}$

Total

$0 / 29$

$0 / 62$

$1 / 349$

$1 / 126$

$0 / 50$

$0 / 22$

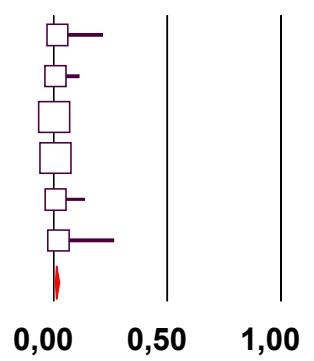

Fig. 6. Complication rate under nucleoplasty for (contained) herniated cervical disc.

a low range. This stands in contrast to the value for control procedures between $0.9 \%$ and $16.2 \%$, which represents an extreme span reaching into the unacceptable. The complication rate under nucleoplasty for herniated cervical discs was $0.8 \%(n=638$ patients $)$ and for herniated lumbar discs $1.8 \%$ ( $n=2,237$ patients) (Figs. 6 and 7).

\section{ODI}

Six studies, 2 of which had control groups, were available for the calculation of ODI values. The nucleoplasty group had a sample size of 318 patients and the control group had 105 patients. All patients underwent lumbar nucleoplasty.
Nucleoplasty showed a significant drop at all time points compared to baseline (58.95), thus revealing improvement in the patients' functional mobility (Table 6).

Fig. 8 shows the ODI values for nucleoplasty as calculated in the meta-analysis in comparison to those for epidural steroid injection, depicted at the measurement time points in the included studies. Nucleoplasty therefore shows a significant decrease and thus improvement in the ODI compared to baseline across all time points. After 3 months and after 6 months, a significantly better ODI can be seen for nucleoplasty than for conservative therapy. All other measured values are also lower, although not significantly. 


\section{Complication rate Nucleoplasty lumbar}

\begin{tabular}{|c|c|c|c|c|}
\hline \multirow[t]{2}{*}{ Study name } & \multicolumn{2}{|c|}{ Statistics for each study } & \multirow[b]{2}{*}{$\begin{array}{l}\text { Upper } \\
\text { limit }\end{array}$} & \multirow[b]{2}{*}{ Total } \\
\hline & $\begin{array}{c}\text { Event } \\
\text { rate }\end{array}$ & $\begin{array}{l}\text { Lower } \\
\text { limit }\end{array}$ & & \\
\hline Azzazi 2011-9 & 0,100 & 0,042 & 0,219 & $5 / 50$ \\
\hline Alexandre $2005-1$ & 0,000 & 0,000 & 0,006 & $0 / 1390$ \\
\hline Bhagia 2006-1 & 0,204 & 0,113 & 0,339 & $10 / 49$ \\
\hline Bokov 2010-9 & 0,007 & 0,000 & 0,099 & $0 / 73$ \\
\hline Calisaneller 2007-4 & 0,017 & 0,001 & 0,217 & $0 / 29$ \\
\hline Gerszten 2006-1 & 0,007 & 0,000 & 0,107 & $0 / 67$ \\
\hline Gerszten 2010-4 & 0,111 & 0,047 & 0,241 & $5 / 45$ \\
\hline Karaman 2011-8 & 0,009 & 0,001 & 0,125 & $0 / 56$ \\
\hline Marin 2005-1 & 0,008 & 0,000 & 0,111 & $0 / 64$ \\
\hline Masala 2007-5 & 0,007 & 0,000 & 0,100 & $0 / 72$ \\
\hline Mirzai 2007-1 & 0,009 & 0,001 & 0,134 & $0 / 52$ \\
\hline Reddy 2005-10 & 0,010 & 0,001 & 0,141 & $0 / 49$ \\
\hline Shabat 2012-8 & 0,006 & 0,000 & 0,084 & $0 / 87$ \\
\hline Sharps 2002-4 & 0,010 & 0,001 & 0,141 & $0 / 49$ \\
\hline Sinan 2011-8 & 0,036 & 0,012 & 0,106 & $3 / 83$ \\
\hline Singh $2002-7$ & 0,007 & 0,000 & 0,107 & $0 / 67$ \\
\hline Singh 2003-7 & 0,006 & 0,000 & 0,091 & $0 / 80$ \\
\hline Singh 2004-10 & 0,010 & 0,001 & 0,146 & $0 / 47$ \\
\hline \multirow{2}{*}{ Yakovlev 2007-3 } & 0,022 & 0,001 & 0,268 & $0 / 22$ \\
\hline & 0,018 & 0,008 & 0,039 & \\
\hline
\end{tabular}

Event rate and $95 \% \mathrm{Cl}$

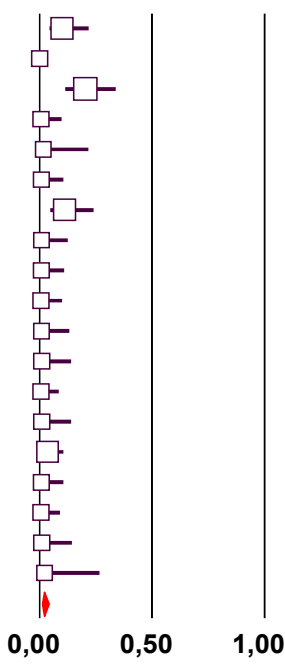

Fig. 7. Complication rate under nucleoplasty for (contained) herniated lumbar disc.

Table 6. Functional mobility after nucleoplasty and after conservative therapy (including epidural steroid injection).

\begin{tabular}{|c|c|c|c|c|}
\hline $\begin{array}{l}\text { Random effects } \\
\text { analysis }\end{array}$ & Patients & ODI nucleoplasty & 95\% CI & $\begin{array}{c}P \text { values in } \\
\text { comparison } \\
\text { to baseline }\end{array}$ \\
\hline Baseline & 318 & 58.95 & {$[45.47 ; 72.43]$} & \\
\hline 1 week & 42 & 28.60 & {$[26.12 ; 31.08]$} & $<0.001$ \\
\hline 2 weeks & 50 & 29.00 & {$[26.26 ; 31.74]$} & $<0.001$ \\
\hline 1 month & 179 & 23.21 & {$[9.33 ; 37.09]$} & $<0.001$ \\
\hline 6 weeks & 40 & 30.00 & {$[24.42 ; 35.58]$} & $<0.001$ \\
\hline 3 months & 153 & 18.30 & {$[8.40 ; 28.19]$} & $<0.001$ \\
\hline 6 months & 256 & 22.54 & {$[10.94 ; 34.13]$} & $<0.001$ \\
\hline 12 months & 264 & 24.43 & {$[13.08 ; 35.79]$} & $<0.001$ \\
\hline 18 months & 73 & 12.82 & {$[9.16 ; 16.47]$} & $<0.001$ \\
\hline \multirow[t]{2}{*}{24 months } & 92 & 36.98 & {$[31.63 ; 42.33]$} & $<0.005$ \\
\hline & Patients & $\begin{array}{c}\text { ODI conservative therapy (including epidural steroid } \\
\text { injection) }\end{array}$ & $95 \%$ CI & $\begin{array}{c}P \text { values in } \\
\text { comparison } \\
\text { to nucleoplasty }\end{array}$ \\
\hline Baseline & 40 & 43 & {$[37.73 ; 48.27]$} & ${ }^{*}<0.05$ \\
\hline 6 weeks & 33 & 38 & {$[33.22 ; 42.78]$} & ${ }^{*}<0.05$ \\
\hline 3 months & 30 & 40 & {$[33.92 ; 46.08]$} & $* 0.001$ \\
\hline 6 months & 28 & 49 & {$[43.44 ; 54.56]$} & ${ }^{*}<0.001$ \\
\hline
\end{tabular}

* Significant improvement of nucleoplasty in comparison to conservative therapy $(P<0.05)$ 


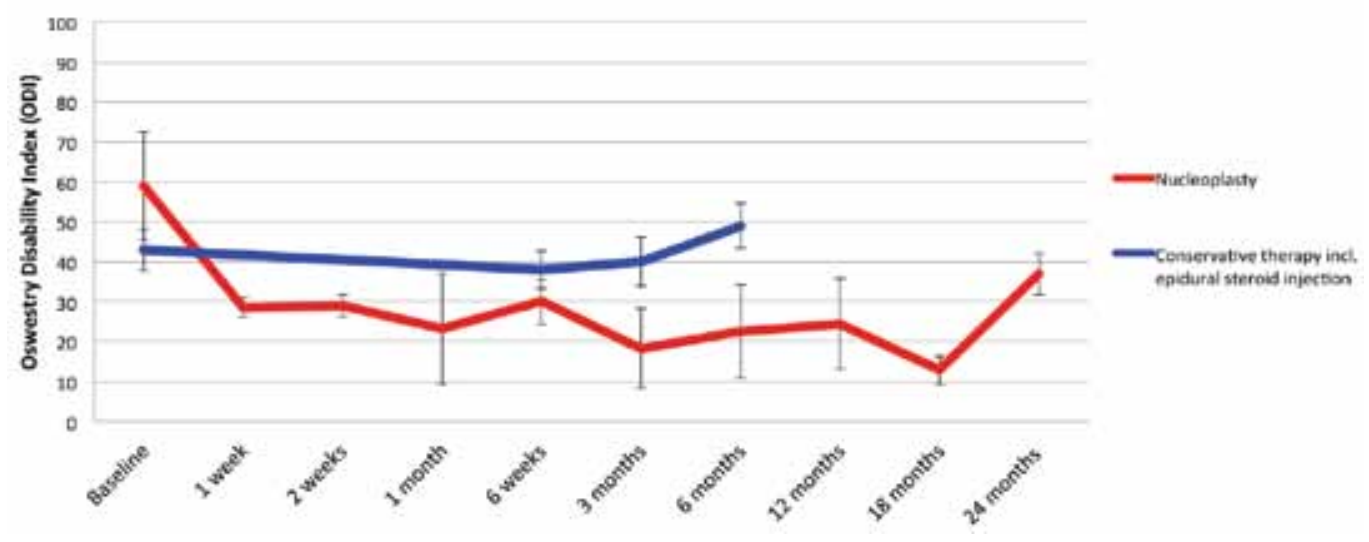

Fig. 8. ODI values after nucleoplasty (lumbar) and conservative therapy.

\section{Discussion}

In the recent past, several reviews on nucleoplasty have appeared; a meta-analysis, however, has not yet been carried out $(17,22,23)$. The review and corresponding meta-analysis presented here provides pooled data on VAS and ODI values as well as an overall complication rate for the selected clinical studies. It was possible to summarize the treatment success of nucleoplasty in previous clinical studies based on the chosen outcome parameters, although we were not able to make a recommendation or evaluation, let alone a catalog of indications. The results, therefore, are conclusions about the effectiveness and safety of the nucleoplasty procedure, as are the intention of a meta-analysis (154).

The studies used in this meta-analysis are heterogeneous for the most part, which is reflected in the varying scores of the studies on the Jadad scale. In order to obtain homogeneity, we applied the Jadad scale to each study and set the minimal score at 2 . This may appear low at first glance, but it can be noted that the included studies contained a specific intervention for a specific indication, namely nucleoplasty for intervertebral disc conditions, with defined measurement time points and intervals suitable for the calculation of a meta-analysis, thus fulfilling the inclusion criteria for this meta-analysis (155).

Particularly for the outcome parameter "complication rate," studies with a low Jadad scale score were also included that were not considered in the analysis of VAS and ODI. Nonetheless, precisely these studies treated patients - successfully - using nucleoplasty, and for that very reason the studies with low Jadad scores were also considered in this meta-analysis. Moreover, one must question whether blinding and randomization in an interventional study on nucleoplasty is necessary at all in order to obtain valid data, since nucleoplasty was also applied successfully even without blinding and randomization.

Setting a low Jadad scale score may reduce the quality of the meta-analysis, yet the meta-analysis should be as meaningful and reliable as possible for the given clinical aspect.

Furthermore, the meta-analysis calculation drew upon a "random effects" model, which takes possible heterogeneity into consideration more than the "fixed effect" model, since the confidence intervals are spread more broadly and thus capture the true value of the meta-analysis. This is therefore a more cautious and conservative estimation, but it can also result in greater inaccuracy through the overestimation of smaller studies and constitute a higher risk for the bias of the (nucleoplasty) results (156).

A (selection) bias could have resulted by excluding the studies by Li et al (108), Fabrizi et al (109), and Theron et al (110). However, the indication for nucleoplasty and the use of Coblation technology, respectively, did not fulfill the corresponding inclusion criterion of the present meta-analysis, namely the clinical application of nucleoplasty for intervertebral disc conditions.

As shown in Table 2, the inclusion and exclusion criteria of the studies were relatively consistent. However, Karaman et al (138) and Mirzai et al (142), for example, excluded patients over 60 years of age from their studies, maintaining that the aging intervertebral disc increasingly dehydrates and undergoes fibrotic changes. 
Interestingly, the study by Sim et al (147) treated 5 patients with nucleoplasty even though contraindications existed according to their exclusion criteria; 2 patients had disc extrusions and 3 patients had spinal stenosis. However, one patient with disc extrusion showed excellent and the other had good results. Of the 3 patients with spinal stenosis, one showed excellent and the other 2 had fair results.

Bokov et al (117) treated 27 patients with disc extrusions. Although the authors concluded that nucleoplasty in these patients was associated with a poor outcome, stable and significant pain relief was observed in $44 \%$ of the patients and even total pain relief in $15 \%$ of the patients. This was another reason to also include patients with disc extrusions in the present meta-analysis.

In the study by Gerszten et al (136), an inclusion criterion for nucleoplasty and for the control group receiving epidural steroid injection was that the patients had already undergone failed epidural steroid injection 3 weeks to 6 months prior to study onset. This could have led to bias of the measurement results especially in the nucleoplasty group, as the patient's psyche may constitute a significant factor in coping with the disease.

In their studies, Singh et al (18-20) excluded all patients who used opioids heavily. Since patients with chronic back pain frequently consume strong opioids for prolonged periods of time, this selection of patients could have led to bias.

Consequently, it cannot be ruled out with certainty that the inclusion and exclusion criteria defined in the included studies caused a possible bias with regard to the patient population and the calculated values for the outcome parameters. Since patients with higher grade spinal degenerations and disc extrusions were included in the studies and consecutive in this meta-analysis, the resulting calculations are likely to be somewhat negatively biased.

\section{VAS}

The application of nucleoplasty results in significant pain reduction at every examination time point compared to baseline, and the patients experienced measurable and, above all, noticeable pain relief.

The need for calculating total VAS values for various areas of pain (e.g., back and leg pain) in patients from the studies by Bhagia et al (132), Gerszten et al (136), Lemcke et al (139), and Zhu et al (150) may have caused biased results. Also, the assumption of a correlation for the study by Bhagia et al (132) and the assump- tion of a standard deviation for the study by Singh et al (19) from their earlier study (18) may also constitute sources of interference, yet they are based on the procedural instructions for meta-analyses as recommended by the Cochrane Collaboration (151).

Karaman et al (138) found that age had no significant effect on VAS values, but a remarkable, negative correlation existed with the duration of pain symptoms.

Lemcke et al (139) observed in their comparative study nucleoplasty vs. "Disc Dekompressor" significantly lower VAS values in the Disc Dekompressor group. The authors attributed this result to lacking randomization and the clearly different symptom durations (nucleoplasty 30.5 months vs. Disc Dekompressor 16 months). Interestingly, in the nucleoplasty group a weak correlation was noted between patient age and outcome, so that younger patients exhibited better results than older patients.

In the study by Bokov et al (117), 3 subgroups were treated with nucleoplasty. Based on the size of the disc protrusion, patients were divided into subgroup 1a or $1 b$. Patients with extrusions were assigned to group 2. In the calculation carried out here, these 3 groups were added together to form one treatment group with pooled VAS data. Since patients with extrusions are actually a contraindication for nucleoplasty, subgroup 2 could have had a negative bias on the total VAS value.

The third patient group was treated using microdiscectomy. Bokov et al (117) concluded from their study results that the size of the disc protrusion does not constitute a predictor of success; in cases of extrusions, however, nucleoplasty yields unsatisfactory results significantly more often compared to microdiscectomy.

In the studies conducted by Singh et al (18-20) from 2002 to 2004, NPS values were documented instead of VAS values, which still lead functionally to analog test results.

Reddy et al (144), Bhagia et al (132), Yakovlev et al (149), Bokov et al (117), and Zhu et al (150) administered a dose of glucocorticoid (mostly betamethasone) and a local anesthetic (mostly lidocaine) immediately after nucleoplasty (in part only to patients with radicular symptoms). This could certainly have had a positive influence on the VAS/NPS as well as the ODI values in the short and medium term.

\section{Complication Rate}

The complication rate for nucleoplasty was $1.5 \%$ ( $0.8 \%$ for cervical, $1.8 \%$ for lumbar nucleoplasty). In no case of nucleoplasty were reports made of severe 
complications such as paraplegia or even death. The low complication rate may most likely be explained by the minimally invasive, percutaneous procedure of nucleoplasty. The unequal complication rates for cervical and lumbar nucleoplasty are most likely due to the different sides of needle entry. An anterior access site is chosen for cervical nucleoplasty, meaning that nerve structures are not passed, and less soft tissue simplifies the depiction of the intervertebral regions.

The complication rate calculated in this metaanalysis includes all events evaluated as complications in the studies. Most studies reported no or no significant complications. If no information pertaining to complications was found, the study had to be deleted from the meta-analysis calculation. It may be assumed, however, that no complications occurred at all, since clinical studies are required to publish any and all occurring complications. Thus, the real complication rate may lie below the rate calculated in this meta-analysis. The following studies demonstrate that the definition of complications was not uniform:

As described previously, the complication rate was adapted for the articles by Bhagia et al (132) and Sinan et al (148).

Bhagia et al (132) conducted a study on side effects and complications that occurred after nucleoplasty. The most important side effects were pain at the puncture site (76\% of patients) and newly appearing back pain $(26 \%)$. At the end of the study after 2 weeks, patients no longer complained about these 2 symptoms. At the middle of the observation period after one week, 14 out of 53 patients noticed newly occurring numbness in the legs which, however, only 10 patients indicated having at the end of the study after 2 weeks. In no case did the numbness cause functional restrictions, and dermatomal mapping of such numbness was not possible, suggesting that the numbness did not involve the compression of a nerve root. Nevertheless, the sensation of numbness was assessed as a complication since the further course was unclear. Due to the short observation period, the later condition of the patients is described insufficiently. Even if the complication rate for this study may possibly seem to be too high in the present meta-analysis, it still appears justifiable to take it into consideration.

Sinan et al (148) stated in their abstract that no complications had been observed. In the results section, however, they reported of 2 patients who had experienced numbness in both legs for 3 months; another patient developed discitis that was able to be treated successfully with spondylodesis. It therefore appears that the authors restricted the description "complications" to hemorrhage, meningitis, and nerve damage. These 3 complications were taken into consideration for the present meta-analysis, since other authors had also described discitis $(127,131,135)$ and numbness as complications (132).

The study by Gerszten et al (136), which compared nucleoplasty with epidural steroid injection, is the only study that describes the complication rate in percent, calls complications "procedure-related adverse events," and defines them very broadly. The complication rate was $11 \%$ in the nucleoplasty group (5 patients with 7 events) and $18 \%$ in the epidural steroid group (7 patients with 14 events). Pain at the puncture site, for example, was evaluated here as an event. This event could be considered self-limiting ( 2 events each per examined group). However, the authors also documented increased radicular pain ( 1 vs. 5 events), increased numbness ( 1 vs. 0 events), increased back pain (1 vs. 4 events), light-headedness ( 0 vs. 1 event), and muscle tightness ( 0 vs. 1 event). Whether these events were self-limiting and how the authors then proceeded with the patients is not mentioned. Since the affected patients were able to report more than one complication, the complication rate could not be lowered in the present meta-analysis.

\section{ODI}

Nucleoplasty shows a significant decrease at all measurement time points compared to baseline and thus an improvement in patients' functional mobility.

Despite the efficacy of epidural steroid injection in cervical (157) and lumbar (158) disc herniations and the cost effectiveness (159) as demonstrated in other studies, at no time did our meta-analysis show significant improvement in control group patients who were treated with conservative therapy including epidural steroid injection. This could be explained by the purely short-term effect of a drug such as glucocorticoids and/ or local anesthetics.

As described above, Reddy et al (144), Bhagia et al (132), Yakovlev et al (149), Bokov et al (117), and Zhu et al (150) administered a dose of glucocorticoid (mostly betamethasone) and local anesthetic (mostly lidocaine) immediately following nucleoplasty. Here, too, the ODI values could surely have been influenced positively in the short and the medium term.

For the same indication reasons stated above, we decided to compare nucleoplasty with conservative therapy. 
The different ODI baseline values in the nucleoplasty group (ODI of 59) compared to the conservative control group (including epidural steroid injection) (ODI of 43) as shown in the subgroup analysis cannot be explained by the inclusion of nonrandomized studies. It seems conceivable that patients with more severe symptoms may be more likely to receive a doctor's recommendation for nucleoplasty.

\section{Conclusions}

Nucleoplasty reduces pain in the long term and increases patients' functional mobility. Compared to other treatments, it is an effective, low-complication, minimally invasive procedure used to treat cervical and lumbar disc herniations. Under the given catalog of indications, it appears to be superior to conservative therapy. Patients experience greater pain relief after cervical nucleoplasty than after lumbar nucleoplasty.

Studies published to date show a heterogeneous picture of inclusion and exclusion criteria. Therefore, a bias of the data presented here cannot be ruled out with certainty. Initial results suggest the possibility of extending the indication to include disc extrusions.

\section{Acknowledgment}

Philipp Maximilian Eichen, MD and Bastian Himpe, $\mathrm{MD}$, contributed equally to this manuscript.

\section{References}

1. Bundesärztekammer (BÄK) KBK. Arbeitsgemeinschaft der Wissenschaftlichen Medizinischen Fachgesellschaften (AWMF). Nationale VersorgungsLeitlinie Kreuzschmerz - Langfassung. 2012; Version 1.2. August 2011, Available from: www.versorgungsleitlinien.de/themen/ kreuzschmerz

2. Schmidt $C O$, Raspe $H$, Pfingsten $M$, Hasenbring M, Basler HD, Eich W, Kohlmann T. Back pain in the German adult population: Prevalence, severity, and sociodemographic correlates in a multiregional survey. Spine (Phila Pa 1976) 2007; 32:2005-2011.

3. Raspe H. Rückenschmerzen - Heft 53. 2012; Available from: www.rki.de/ $\mathrm{DE} /$ Content/Gesundheitsmonitoring/Gesundheitsberichterstattung/ GBEDownloadsT/rueckenschmerzen. pdf?_blob=publicationFile

4. Latza U, Kohlmann T, Deck R, Raspe H. Influence of occupational factors on the relation between socioeconomic status and self-reported back pain in a population-based sample of German adults with back pain. Spine (Phila Pa 1976) 2000; 25:1390-1397.

5. Bethge M. [Patients with low back pain. Psychosocial work-related factors and return to work - a literature review]. Orthopade 2010; 39:866-873.

6. Hartvigsen J, Leboeuf-Yde C, Lings S, Corder E.H. Is sitting-while-at-work associated with low back pain? A systematic, critical literature review. Scand J Public Health 2000; 28:230-239.

7. Deyo RA, Mirza SK, Martin BI. Back pain prevalence and visit rates: Estimates from U.S. national surveys, 2002. Spine
(Phila Pa 1976) 2006; 31:2724-2727.

8. Fogarty International Center of the U.S National Institutes of Health, T.W.B. World Health Organization, Population Reference Bureau, Foundation, B.M.G. Disease Control Priorities Project - Musculoskeletal conditions are the most common cause of chronic disability. 2007; Available from: www.dcp2.org/ file/84/DCPP-Musculoskeletal.pdf.

9. WHO. Disability-adjusted life year (DALY) rates. 25.08.2013; Available from: www. who.int/gho/mortality_burden_disease/ daly_rates/text/en/.

10. Institute of Medicine Committee on Advancing Pain Research, C.a.E., The National Academies Collection: Reports funded by National Institutes of Health, in Relieving Pain in America: A Blueprint for Transforming Prevention, Care, Education, and Research. National Academies Press, National Academy of Sciences, Washington DC, 2011.

11. Gaskin DJ, Richard P. The economic costs of pain in the United States. J Pain 2012; 13:715-724.

12. Pain Proposal Consensus Committee. Pain Proposal. 2010; Available from: www.dgss.org/fileadmin/pdf/Pain_Proposal_European_Consensus_Report. pdf.

13. Manchikanti L, Abdi S, Atluri S, Benyamin RM, Boswell MV, Buenaventura RM, Bryce DA, Burks PA, Caraway DL, Calodney AK, Cash KA, Christo PJ, Cohen SP, Colson J, Conn A, Cordner H, Coubarous S, Datta S, Deer TR, Diwan S, Falco FJ, Fellows B, Geffert S, Grider JS, Gupta S, Hameed H, Hameed M, Hansen $H$, Helm S, Janata JW, Justiz R, Kaye
AD, Lee M, Manchikanti KN, McManus CD, Onyewu O, Parr AT, Patel VB, Racz GB, Sehgal N, Sharma ML, Simopoulos TT, Singh V, Smith HS, Snook LT, Swicegood JR, Vallejo R, Ward SP, Wargo BW, Zhu J, Hirsch JA. An update of comprehensive evidence-based guidelines for interventional techniques in chronic spinal pain. Part II: Guidance and recommendations. Pain Physician 2013; 16:S49-S283.

14. Theodoridis T. [Injection therapy of the spine without imaging]. Orthopade 2007; 36:73-85; quiz 86.

15. Weinstein JN, Lurie JD, Tosteson TD, Tosteson AN, Blood EA, Abdu WA, Herkowitz $\mathrm{H}$, Hilibrand $\mathrm{A}$, Albert $\mathrm{T}$, Fischgrund J. Surgical versus nonoperative treatment for lumbar disc herniation: Four-year results for the Spine Patient Outcomes Research Trial (SPORT). Spine (Phila Pa 1976) 2008; 33:2789-2800.

16. Krämer J, Ludwig J. [Surgical treatment of lumbar intervertebral disk displacement. Indications and methods]. Orthopade 1999; 28:579-584.

17. Gerges FJ, Lipsitz SR, Nedeljkovic SS. A systematic review on the effectiveness of the nucleoplasty procedure for discogenic pain. Pain Physician 2010; 13:117-132.

18. Singh V, Piryani C, Liao K, Nieschulz S. Percutaneous disc decompression using coblation (nucleoplasty) in the treatment of chronic discogenic pain. Pain Physician 2002; 5:250-259.

19. Singh V, Piryani C, Liao K. Evaluation of percutaneous disc decompression using coblation in chronic back pain with or without leg pain. Pain Physician 2003; 
6:273-280.

20. Singh V, Piryani C, Liao K. Role of percutaneous disc decompression using coblation in managing chronic discogenic low back pain: A prospective, observational study. Pain Physician 2004; 7:419-425.

21. König V, Eichen PM, Achilles N, Mösges R. A systematic review of RCTs with nucleoplasty - an update. Pain Physician 2013; 16:E45-E46.

22. Manchikanti L, Falco FJ, Benyamin RM, Caraway DL, Deer TR, Singh V, Hameed $\mathrm{H}$, Hirsch JA. An update of the systematic assessment of mechanical lumbar disc decompression with nucleoplasty. Pain Physician 2013; 16:SE25-SE54.

23. Manchikanti L, Derby R, Benyamin RM, Helm S, Hirsch JA. A systematic review of mechanical lumbar disc decompression with nucleoplasty. Pain Physician 2009; 12:561-572.

24. Ressing M, Blettner M, Klug SJ. Systematic literature reviews and meta-analyses: Part 6 of a series on evaluation of scientific publications. Dtsch Arztebl Int 2009; 106:456-463.

25. Knop C, Oeser M, Bastian L, Lange U, Zdichavsky M, Blauth M. [Development and validation of the Visual Analogue Scale (VAS) Spine Score]. Unfallchirurg 2001; 104:488-497.

26. Fairbank JC, Couper J, Davies JB, O'Brien JP. The Oswestry low back pain disability questionnaire. Physiotherapy 1980; 66:271-273.

27. Juni P, Holenstein F, Sterne J, Bartlett C, Egger M. Direction and impact of language bias in meta-analyses of controlled trials: Empirical study. Int J Epidemiol 2002; 31:115-123.

28. Birnbaum K. Percutaneous cervical disc decompression. Surg Radiol Anat 2009; 31:379-387.

29. Marín FZ. CAM versus nucleoplasty. Acta Neurochir Suppl 2005; 92:111-114.

30. Gerszten PC, Welch WC, McGrath PM, Willis SL. A prospective outcomes study of patients undergoing intradiscal electrothermy (IDET) for chronic low back pain. Pain Physician 2002; 5:360-364.

31. Biyani A, Andersson GB, Chaudhary H, An HS. Intradiscal electrothermal therapy: A treatment option in patients with internal disc disruption. Spine (Phila Pa 1976) 2003; 28:S8-14.

32. Lee MS, Cooper G, Lutz GE, Lutz C, Hong HM. Intradiscal electrothermal therapy (IDET) for treatment of chronic lumbar discogenic pain: A minimum 2-year clinical outcome study. Pain Physician 2003; 6:443-448.

33. Rosen S, Falco, F. Radiofrequency stimulation of intervertebral discs. Pain Physician 2003; 6:435-438.

34. Freedman BA, Cohen SP, Kuklo TR, Lehman, RA, Larkin P, Giuliani JR. Intradiscal electrothermal therapy (IDET) for chronic low back pain in active-duty soldiers: 2-year follow-up. Spine ] 2003; 3:502-509.

35. Freeman BJ, Walters RM, Moore RJ, Fraser RD. Does intradiscal electrothermal therapy denervate and repair experimentally induced posterolateral annular tears in an animal model? Spine (Phila Pa 1976) 2003; 28:2602-2608.

36. Derby R, Eek B, Lee SH, Seo KS, Kim BJ. Comparison of intradiscal restorative injections and intradiscal electrothermal treatment (IDET) in the treatment of low back pain. Pain Physician 2004; 7:63-66.

37. Pauza KJ, Howell S, Dreyfuss P, Peloza JH, Dawson K, Bogduk N. A randomized, placebo-controlled trial of intradiscal electrothermal therapy for the treatment of discogenic low back pain. Spine J 2004; 4:27-35.

38. Derby R, Chen Y, Lee SH, Seo KS, Kim $B$ B. Non-surgical interventional treatment of cervical and thoracic radiculopathies. Pain Physician 2004; 7:389-394.

39. Tsou PM, Alan Yeung C, Yeung AT. Posterolateral transforaminal selective endoscopic discectomy and thermal annuloplasty for chronic lumbar discogenic pain: A minimal access visualized intradiscal surgical procedure. Spine ] 2004; 4:564-573.

40. Derby R, Lee SH, Seo KS, Kazala K, Kim BJ, Kim MJ. Efficacy of IDET for relief of leg pain associated with discogenic low back pain. Pain Pract 2004; 4:281-285.

41. Lühmann D, Burkhardt-Hammer T, Borowski C, Raspe H. Minimalinvasive Verfahren zur Behandlung des Bandscheibenvorfalles. 2005; 1. Auflage: Available from: http://portal.dimdi.de/de/hta/hta_berichte/ hta108_bericht_de.pdf.

42. Pollintine P, Findlay G, Adams MA. Intradiscal electrothermal therapy can alter compressive stress distributions inside degenerated intervertebral discs. Spine (Phila Pa 1976) 2005; 30:E134-139.

43. Derby R, Seo KS, Kazala K, Chen YC, Lee $\mathrm{SH}$, Kim BJ. A factor analysis of lumbar intradiscal electrothermal annuloplasty outcomes. Spine J 2005; 5:256-261; discussion 262.
44. Freeman BJ, Fraser RD, Cain CM, Hall DJ, Chapple DC. A randomized, doubleblind, controlled trial: Intradiscal electrothermal therapy versus placebo for the treatment of chronic discogenic low back pain. Spine (Phila Pa 1976) 2005; 30:2369-2377; discussion 2378.

45. Kapural L, Hayek S, Malak O, Arrigain S, Mekhail N. Intradiscal thermal annuloplasty versus intradiscal radiofrequency ablation for the treatment of discogenic pain: A prospective matched control trial. Pain Med 2005; 6:425-431.

46. Münnich $U$, Kreckel V, Koy T, König DP, Heyll U. [Intradiscal electrothermal therapy (IDET)]. Versicherungsmedizin 2005; 57:182-184.

47. Southern D, Lutz G, Bracilovic A, West P, Spevak M, Camacho NP, Doty S. Histological and molecular structure characterization of annular collagen after intradiskal electrothermal annuloplasty. HSS ] 2006; 2:49-54.

48. Park SY, Moon SH, Park MS, Kim HS, Choi YJ, Lee HM. Intradiscal electrothermal treatment for chronic lower back pain patients with internal disc disruption. Yonsei Med J 2005; 46:539-545.

49. Rozen D, Grass, GW. Intradiscal electrothermal coagulation and percutaneous neuromodulation therapy in the treatment of discogenic low back pain. Pain Pract 2005; 5:228-243.

50. Singh K, Ledet E, Carl A. Intradiscal therapy: A review of current treatment modalities. Spine (Phila Pa 1976) 2005; 30:S2O-26.

51. Chen F, Lü G, Kang Y, Ma Z, Lu C, Wang B, Li J, Liu J, Li H. Mucormycosis spondylodiscitis after lumbar disc puncture. Eur Spine J 2006; 15:370-376.

52. Zhou Y, Furgang FA, Zhang Y. Quality assurance for interventional pain management procedures. Pain Physician 2006; 9:107-114.

53. Fukui S. Changes on MRI in lumbar disc protrusions in two patients after intradiscal electrothermal therapy. J Anesth 2006; 20:132-134.

54. Andersson GB, Mekhail NA, Block JE. Treatment of intractable discogenic low back pain. A systematic review of spinal fusion and intradiscal electrothermal therapy (IDET). Pain Physician 2006; 9:237-248.

55. Appleby D, Andersson G, Totta M. Metaanalysis of the efficacy and safety of intradiscal electrothermal therapy (IDET). Pain Med 2006; 7:308-316.

56. Freeman BJ. IDET: A critical appraisal of 
the evidence. Eur Spine J 2006; 15 Suppl 3:S448-S457.

57. Urrútia G, Kovacs F, Nishishinya MB, Olabe J. Percutaneous thermocoagulation intradiscal techniques for discogenic low back pain. Spine (Phila Pa 1976) 2007; 32:1146-1154.

58. Basaran A, Topatan S. Spinal balloon nucleoplasty: A hypothetical minimally invasive treatment for herniated nucleus pulposus. Med Hypotheses 2008; 70:1201-1206.

59. Durán Sacristán H. [Lumbago and sciatica]. An R Acad Nac Med (Madr) 2008; 125:403-422; discussion 423-405.

6o. Jawahar A, Brandao SM, Howard C, Nunley PD. Intradiscal electrothermal therapy (IDET): A viable alternative to surgery for low back pain in workers' compensation patients? J La State Med Soc 2008; 160:280-285.

61. Kloth DS, Fenton DS, Andersson GB, Block JE. Intradiscal electrothermal therapy (IDET) for the treatment of discogenic low back pain: Patient selection and indications for use. Pain Physician 2008; 11:659-668.

62. Nunley PD, Jawahar A, Brandao SM, Wilkinson KM. Intradiscal electrothermal therapy (IDET) for low back pain in worker's compensation patients: Can it provide a potential answer? Longterm results. J Spinal Disord Tech 2008; 21:11-18.

63. Maurer P, Block JE, Squillante D. Intradiscal electrothermal therapy (IDET) provides effective symptom relief in patients with discogenic low back pain. J Spinal Disord Tech 2008; 21:55-62.

64. Manchikanti L, Singh V, Derby $R$, Schultz DM, Benyamin RM, Prager JP, Hirsch JA. Reassessment of evidence synthesis of occupational medicine practice guidelines for interventional pain management. Pain Physician 2008; 11:393-482.

65. Manchikanti L, Singh V, Derby R, Helm $S$, Trescot AM, Staats PS, Prager JP, Hirsch JA. Review of occupational medicine practice guidelines for interventional pain management and potential implications. Pain Physician 2008; 11:271-289.

66. Levin JH. Prospective, double-blind, randomized placebo-controlled trials in interventional spine: What the highest quality literature tells us. Spine ] 2009; 9:690-703.

67. Jasper JF. ACOEM guideline analysis. Pain Physician 2008; 11:701-703; author reply 705-706.
68. Brown LL, Escobar LA, Stein A. Comment on Manchikanti et al's ACOEM guideline criticism. Pain Physician 2008; 11:700; author reply 705-706.

69. Shchedrenok VV, Ivanenko AV, Sebelev KI, Moguchaia OV, Zevakhin SV. [Use of a diode laser in surgery of intervertebral disks in osteochondrosis of the vertebral column]. Vestn Khir Im I I Grek 2009; 168:42-43.

70. Helm S, Hayek SM, Benyamin RM, Manchikanti L. Systematic review of the effectiveness of thermal annular procedures in treating discogenic low back pain. Pain Physician 2009; 12:207-232.

71. Manchikanti L, Shah RV, Datta S, Singh, V. Critical evaluation of interventional pain management literature provides inaccurate conclusions. Spine J 2009; 9:706-708; author reply 708-709.

72. Chou R. Same trials, different conclusions: Sorting out discrepancies between reviews on interventional procedures of the spine. Spine J 2009; 9:679-689.

73. Kara B, Yildirim Y, Erbayraktar S. Physiotherapy results after nucleoplasty. Neurosciences (Riyadh) 2009; 14:389-390.

74. Klimenko K, Vishniakov VV. [The results of the surgical treatment of obstructive sleep apnea syndrome with the use of the cold plasma technique]. Vestn Otorinolaringol 2011:44-46.

75. Welch WC, Gerszten PC. Alternative strategies for lumbar discectomy: Intradiscal electrothermy and nucleoplasty. Neurosurg Focus 2002; 13:E7.

76. Dworkin GE. Advanced concepts in interventional spine care. J Am Osteopath Assoc 2002; 102:S8-S11.

77. Andreula C, Muto M, Leonardi, M. Interventional spinal procedures. Eur] Radiol 2004; 50:112-119.

78. Anderson PA, Rouleau JP. Intervertebral disc arthroplasty. Spine (Phila Pa 1976) 2004; 29:2779-2786.

79. Erdine S, Ozyalçin NS, Cimen A. [Percutaneous lumber nucleoplasty]. Agri 2005; 17:17-22.

80. Crawford NR. Biomechanics of lumbar arthroplasty. Neurosurg Clin N Am 2005; 16:595-602, v.

81. Singh V, Derby R. Percutaneous lumbar disc decompression. Pain Physician 2006; 9:139-146.

82. Pomerantz SR, Hirsch JA. Intradiscal therapies for discogenic pain. Semin Musculoskelet Radiol 2006; 10:125-135.

83. Nezer D, Hermoni D. [Percutaneous dis- cectomy and intradiscal radiofrequency thermocoagulation for low back pain: Evaluation according to the best available evidence]. Harefuah 2007; 146:747750, 815.

84. Freeman BJ, Mehdian R. Intradiscal electrothermal therapy, percutaneous discectomy, and nucleoplasty: What is the current evidence? Curr Pain Headache Rep 2008; 12:14-21.

85. Knight K, Woods DM, McHaourab A. Nucleoplasty for disc protrusion: A novel percutaneous decompression technique. Techniques in Regional Anesthesia Q Pain Management 2009; 13:93-101.

86. Chou R, Atlas SJ, Stanos SP, Rosenquist RW. Nonsurgical interventional therapies for low back pain: A review of the evidence for an American Pain Society clinical practice guideline. Spine (Phila $\mathrm{Pa}$ 1976) 2009; 34:1078-1093.

87. Gangi A, Tsoumakidou G, Buy X, Cabral JF, Garnon J. Percutaneous techniques for cervical pain of discal origin. Semin Musculoskelet Radiol 2011; 15:172-180.

88. Gebremariam L, Koes BW, Peul WC, Huisstede BM. Evaluation of treatment effectiveness for the herniated cervical disc: A systematic review. Spine (Phila Pa 1976) 2012; 37:E109-E118.

89. Lee SC. Percutaneous intradiscal treatments for discogenic pain. Acta Anaesthesiol Taiwan 2012; 50:25-28.

90. Grewal H, Grewal BS, Patel R. Nonsurgical interventions for low back pain. Prim Care 2012; 39:517-523.

91. Chen YC, Lee SH, Chen D. Intradiscal pressure study of percutaneous disc decompression with nucleoplasty in human cadavers. Spine (Phila Pa 1976) 2003; 28:661-665.

92. Lee MS, Cooper G, Lutz GE, Doty SB. Histologic characterization of coblation nucleoplasty performed on sheep intervertebral discs. Pain Physician 2003; 6:439-442.

93. Chen YC, Lee SH, Saenz Y, Lehman NL. Histologic findings of disc, end plate and neural elements after coblation of nucleus pulposus: An experimental nucleoplasty study. Spine ] 2003; 3:466-470.

94. O’Neill CW, Liu JJ, Leibenberg E, Hu SS, Deviren V, Tay BK, Chin CT, Lotz JC. Percutaneous plasma decompression alters cytokine expression in injured porcine intervertebral discs. Spine J 2004; 4:88-98.

95. Nau WH, Diederich CJ. Evaluation of temperature distributions in cadaveric lumbar spine during nucleoplasty. Phys 
Med Biol 2004; 49:1583-1594.

96. Wang JC, Kabo JM, Tsou PM, Halevi L, Shamie AN. The effect of uniform heating on the biomechanical properties of the intervertebral disc in a porcine model. Spine J 2005; 5:64-70.

97. Wang Y, Deng Y. [Signaling of biglycan and cytokines in bovine intervertebral disc cells]. Zhong Nan Da Xue Xue Bao Yi Xue Ban 2011; 36:889-894.

98. Sato $M$, Ishihara $M$, Kikuchi M, Mochida $J$. The influence of Ho:YAG laser irradiation on intervertebral disc cells. Lasers Surg Med 2011; 43:921-926.

99. Kasch R, Mensel B, Schmidt F, Drescher W, Pfuhl R, Ruetten S, Merk HR, Kayser R. Percutaneous disc decompression with nucleoplasty-volumetry of the nucleus pulposus using ultrahigh-field MRI. PLoS One 2012; 7:e41497.

100. Kasch R, Mensel B, Schmidt F, Ruetten $S$, Barz T, Froehlich S, Seipel R, Merk $H R$, Kayser R. Disc volume reduction with percutaneous nucleoplasty in an animal model. PLoS One 2012; 7:e50211.

101. Boswell MV, Shah RV, Everett CR, Sehgal N, McKenzie Brown AM, Abdi S, Bowman RC, Deer TR, Datta S, Colson JD, Spillane WF, Smith HS, Lucas LF, Burton AW, Chopra P, Staats PS, Wasserman RA, Manchikanti L. Interventional techniques in the management of chronic spinal pain: Evidence-based practice guidelines. Pain Physician 2005; 8:1-47.

102. Boswell MV, Trescot AM, Datta S, Schultz DM, Hansen HC, Abdi S, Sehgal N, Shah RV, Singh V, Benyamin RM, Patel VB, Buenaventura RM, Colson JD, Cordner HJ, Epter RS, Jasper JF, Dunbar EE, Atluri SL, Bowman RC, Deer TR, Swicegood JR, Staats PS, Smith HS, Burton AW, Kloth DS, Giordano J, Manchikanti L. ASIPP, Interventional techniques: Evidence-based practice guidelines in the management of chronic spinal pain. Pain Physician 2007; 10:7-111.

103. Kallewaard JW, Terheggen MA, Groen G), Sluijter ME, Derby R, Kapural L, Mekhail N, van Kleef M. 15. Discogenic low back pain. Pain Pract 2010; 10:560-579.

104. Mayer HM. Reviewer's comment concerning "Percutaneous cervical nucleoplasty treatment in the cervical disc herniation" (Jian Li et al. MS-no: ESJO-D08-00079R2). Eur Spine J 2008; 17:1670.

105. Li J, Shen H, Hou T, Li M, He S, Zhang $\mathrm{H}$. Acquired hemophilia a in a patient with lumbar disc herniation: A case report and review of the literature. Spine (Phila Pa 1976) 2009; 34:E305-E308.
106. Zhu H, Zhou XZ, Cheng MH, Luo ZP, Ai $H Z$. Coblation nucleoplasty for adjacent segment degeneration after posterolateral fusion surgery: A case report. J Back Musculoskelet Rehabil 2012; 25:235-238.

107. Cuellar VG, Cuellar JM, Vaccaro AR, Carragee EJ, Scuderi GJ. Accelerated degeneration after failed cervical and lumbar nucleoplasty. J Spinal Disord Tech 2010; 23:521-524.

108. Li J, Yan D, Duan L, Zhang Z, Zhu H. Percutaneous discectomy and drainage for postoperative intervertebral discitis. Arch Orthop Trauma Surg 2011; 131:173-178.

109. Fabrizi AP, Zucchelli M. Surgical intradiscal decompression without annulotomy in lumbar disc herniation using a coblation device: Preliminary results. Acta Neurochir Suppl 2005; 92:107-109.

110. Theron J, Guimaraens L, Casasco A, Sola $\mathrm{T}$, Cuellar H, Courtheoux P. Percutaneous treatment of lumbar intervertebral disk hernias with radiopaque gelified ethanol: A preliminary study. J Spinal Disord Tech 2007; 20:526-532.

111. Li J, Yan DL, Gao LB, Tan PX, Zhang ZH, Zhang Z. [Comparison percutaneous cervical disc nucleoplasty and cervical discectomy for the treatment of cervical disc herniation]. Zhonghua Wai Ke Za Zhi 2006; 44:822-825.

112. Zakirov AA, Dreval' ON, Chagava DA, Rynkov IP, Kuznetsov AV. [Treatment of spondyloarthrosis and lumbar discopathy by combined minimally invasive techniques]. Zh Vopr Neirokhir Im N N Burdenko 2012; 76:17-22; discussion 22.

113. Manukovski VA, Badalov VI, Tiulikov $\mathrm{KV}$, Korostelev KE. [Coblation of nucleus pulposus in treatment of military men's lumbar disc protrusions]. Voen Med $\mathrm{Zh}$ 2012; 333:28-34.

114. Cohen SP, Williams S, Kurihara C, Griffith S, Larkin TM. Nucleoplasty with or without intradiscal electrothermal therapy (IDET) as a treatment for lumbar herniated disc. J Spinal Disord Tech 2005; 18:S119-S124.

115. Yan D, Li J, Zhu H, Zhang Z, Duan L. Percutaneous cervical nucleoplasty and percutaneous cervical discectomy treatments of the contained cervical disc herniation. Arch Orthop Trauma Surg 2010; 130:1371-1376.

116. Bokov A, Isrelov A, Skorodumov A, Aleynik A, Simonov A, Mlyavykh S. An analysis of reasons for failed back surgery syndrome and partial results after different types of surgical lumbar nerve root decompression. Pain Physician
2011; 14:545-557.

117. Bokov A, Skorodumov A, Isrelov A, Stupak Y, Kukarin A. Differential treatment of nerve root compression pain caused by lumbar disc herniation applying nucleoplasty. Pain Physician 2010; 13:469-480.

118. Singh V. Percutaneous disc decompression for the treatment of chronic atypical cervical discogenic pain. Pain Physician 2004; 7:115-118.

119. Smuck M, Benny B, Han A, Levin J. Epidural fibrosis following percutaneous disc decompression with coblation technology. Pain Physician 2007; 10:691-696.

120. Puentedura EJ, Brooksby CL, Wallmann HW, Landers MR. Rehabilitation following lumbosacral percutaneous nucleoplasty: A case report. J Orthop Sports Phys Ther 2010; 40:214-224.

121. Pace C, Reyna J, Schlicht C. Percutaneous disc decompression for the treatment of lumbar spinal stenosis. Pain Physician 2003; 6:509-512.

122. Chua NH, Gültuna I, Riezebos P, Beems T, Vissers KC. Percutaneous thoracic intervertebral disc nucleoplasty: Technical notes from 3 patients with painful thoracic disc herniations. Asian Spine J 2011; 5:15-19.

123. Group, O.L.o.E.W. The Oxford Levels of Evidence 2. 2011; Available from: www. cebm.net/index.aspx?o $=5653$.

124. Reverberi C, Bottoli MG, Pennini M, Gabba E. Disc coablation and epidural injection of steroids: A comparison of strategies in the treatment of mechanical spinal discogenic pain. Acta Neurochir Suppl 2005; 92:127-128.

125. Al-Zain F, Lemcke J, Killeen T, Meier U, Eisenschenk A. Minimally invasive spinal surgery using nucleoplasty: A 1-year follow-up study. Acta Neurochir (Wien) 2008; 150:1257-1262.

126. Wolter T, Mohadjer M, Knoeller SM, Berlis A. CT-guided nucleoplasty: Preliminary experience. Klin Neuroradiol 2009; 19:153-156.

127. Bonaldi G, Baruzzi F, Facchinetti A, Fachinetti $P$, Lunghi $S$. Plasma radio-frequency-based diskectomy for treatment of cervical herniated nucleus pulposus: feasibility, safety, and preliminary clinical results. AJNR Am J Neuroradiol 2006; 27:2104-2111.

128. Jadad AR, Moore RA, Carroll D, Jenkinson C, Reynolds DJ, Gavaghan DJ, McQuay HJ. Assessing the quality of reports of randomized clinical trials: Is blinding necessary? Control Clin Trials 1996; 17:1-12. 
129. Oremus $M$, Wolfson C, Perrault A, Demers L, Momoli F, Moride Y. Interrater reliability of the modified Jadad quality scale for systematic reviews of Alzheimer's disease drug trials. Dement Geriatr Cogn Disord 2001; 12:232-236.

130. Alexandre A, Corò L, Azuelos A, Pellone M. Percutaneous nucleoplasty for discoradicular conflict. Acta Neurochir Suppl 2005; 92:83-86.

131. Azzazi A, Al Mekawi S, Zein M. Lumbar disc nucleoplasty using coblation technology: Clinical outcome. J Neurointerv Surg 2011; 3:288-292.

132. Bhagia SM, Slipman CW, Nirschl M, Isaac Z, El-Abd O, Sharps LS, Garvin C. Side effects and complications after percutaneous disc decompression using coblation technology. Am J Phys Med Rehabil 2006; 85:6-13.

133. Calisaneller T, Ozdemir O, Karadeli E, Altinors N. Six months post-operative clinical and 24 hour post-operative MRI examinations after nucleoplasty with radiofrequency energy. Acta Neurochir (Wien) 2007; 149:495-500; discussion 500.

134. Cesaroni A, Nardi PV. Plasma disc decompression for contained cervical disc herniation: A randomized, controlled trial. Eur Spine J 2010; 19:477-486.

135. Cesaroni A, Nardi PV. Plasma-mediated disc decompression for contained cervical disc herniation: Results through 5 years. Acta Neurochir Suppl 2011; 108:113-116.

136. Gerszten PC, Smuck M, Rathmell JP, Simopoulos TT, Bhagia SM, Mocek CK, Crabtree T, Bloch DA, Group SS. Plasma disc decompression compared with fluoroscopy-guided transforaminal epidural steroid injections for symptomatic contained lumbar disc herniation: A prospective, randomized, controlled trial. J Neurosurg Spine 2010; 12:357-371.

137. Gerszten PC, Welch WC, King JT. Quality of life assessment in patients undergoing nucleoplasty-based percutaneous discectomy. J Neurosurg Spine 2006; 4:36-42.

138. Karaman $H$, Tüfek A, Ölmez Kavak $G$, Yildirim ZB, Temel V, Çelik F, Akdemir MS, Kaya S. Effectiveness of nucleoplasty applied for chronic radicular pain. Med Sci Monit 2011; 17:CR461-CR466.

139. Lemcke J, Al-Zain F, Mutze S, Meier U. Minimally invasive spinal surgery using nucleoplasty and the Dekompressor tool: A comparison of two methods in a one year follow-up. Minim Invasive Neurosurg 2010; 53:236-242.

140. Li J, Yan DL, Zhang ZH. Percutaneous cervical nucleoplasty in the treatment of cervical disc herniation. Eur Spine ] 2008; 17:1664-1669.

141. Masala S, Massari F, Fabiano S, Ursone A, Fiori R, Pastore F, Simonetti G. Nucleoplasty in the treatment of lumbar diskogenic back pain: One year followup. Cardiovasc Intervent Radiol 2007; 30:426-432.

142. Mirzai H, Tekin I, Yaman O, Bursali A. The results of nucleoplasty in patients with lumbar herniated disc: A prospective clinical study of 52 consecutive patients. Spine ] 2007; 7:88-92; discussion 92-83.

143. Nardi PV, Cabezas D, Cesaroni A. Percutaneous cervical nucleoplasty using coblation technology. Clinical results in fifty consecutive cases. Acta Neurochir Suppl 2005; 92:73-78.

144. Reddy AS, Loh S, Cutts J, Rachlin J, Hirsch JA. New approach to the management of acute disc herniation. Pain Physician 2005; 8:385-390.

145. Shabat S, David R, Folman Y. Nucleoplasty is effective in reducing both mechanical and radicular low back pain: A prospective study in 87 patients. J Spinal Disord Tech 2012; 25:329-332.

146. Sharps LS, Isaac Z. Percutaneous disc decompression using nucleoplasty. Pain Physician 2002; 5:121-126.

147. Sim SE, Ko ES, Kim DK, Kim HK, Kim YC, Shin HY. The results of cervical nucleoplasty in patients with cervical disc disorder: A retrospective clinical study of 22 patients. Korean J Pain 2011; 24:36-43.

148. Sinan T, Sheikh M, Buric J, Dashti K, AlMukhaimi A. Percutaneous coblation nucleoplasty in patients with contained lumbar disc prolapse: 1 year follow-up in a prospective case series. Acta Neurochir Suppl 2011; 108:107-112.

149. Yakovlev A, Tamimi MA, Liang H, Eristavi M. Outcomes of percutaneous disc decompression utilizing nucleoplasty for the treatment of chronic discogenic pain. Pain Physician 2007; 10:319-328.

150. Zhu H, Zhou XZ, Cheng MH, Shen YX, Dong QR. The efficacy of coblation nucleoplasty for protrusion of lumbar intervertebral disc at a two-year follow-up. Int Orthop 2011; 35:1677-1682.

151. Higgins JP, Green S. Cochrane Handbook for Systematic Reviews of Inter- ventions Version 5.1.0 [updated March 2011]. The Cochrane Collaboration, 2008, 2011.

152. Jacobs WC, van Tulder M, Arts M, Rubinstein SM, van Middelkoop M, Ostelo $R$, Verhagen $A$, Koes $B$, Peul WC. Surgery versus conservative management of sciatica due to a lumbar herniated disc: A systematic review. Eur Spine J 2011; 20:513-522.

153. Kovacs FM, Urrútia G, Alarcón JD. Surgery versus conservative treatment for symptomatic lumbar spinal stenosis: A systematic review of randomized controlled trials. Spine (Phila Pa 1976) 2011; 36:E1335-E1351.

154. Timmer A, Richter B. Systematische Übersichtsarbeiten zu Fragen der Therapie und Prävention Eine Einführung in Frage und Antwort Teil 1 - Was ist eine systematische Übersichtsarbeit? Arzneimitteltherapie 2008; 26:137-139.

155. Timmer A, Richter B. Systematische Übersichtsarbeiten zu Fragen der Therapie und Prävention Eine Einführung in Frage und Antwort Teil 2 - Was macht eine gute Übersichtsarbeit aus? Arzneimitteltherapie 2008; 26:252-255.

156. Timmer A, Rücker XG. Systematische Übersichtsarbeiten zu Fragen der Therapie und Prävention Eine Einführung in Frage und Antwort Teil 3-Wie werden die Ergebnisse zusammengefasst und dargestellt? Arzneimitteltherapie 2008; 26:299-303.

157. Manchikanti L, Cash KA, Pampati V Wargo BW, Malla Y. A randomized, double-blind, active control trial of fluoroscopic cervical interlaminar epidural injections in chronic pain of cervical disc herniation: Results of a 2-year follow-up. Pain Physician 2013; 16:465-478.

158. Manchikanti L, Cash KA, McManus CD, Pampati $\mathrm{V}$, Benyamin RM. A randomized, double-blind, active-controlled trial of fluoroscopic lumbar interlaminar epidural injections in chronic axial or discogenic low back pain: Results of 2-year follow-up. Pain Physician 2013; 16:E491-E504.

159. Manchikanti L, Falco FJ, Pampati V, Cash KA, Benyamin RM, Hirsch JA. Cost utility analysis of caudal epidural injections in the treatment of lumbar disc herniation, axial or discogenic low back pain, central spinal stenosis, and post lumbar surgery syndrome. Pain Physician 2013; 16:E129-E143. 
